\title{
IMPULSIVE STOCHASTIC FUNCTIONAL DIFFERENTIAL INCLUSIONS DRIVEN BY A FRACTIONAL BROWNIAN MOTION WITH INFINITE DELAY
}

\author{
AHMED BOUDAOUI, TOMÁS CARABALLO, AND ABDELGHANI OUAHAB
}

\begin{abstract}
In this paper, we prove the existence of mild solutions for the following first-order impulsive semilinear stochastic functional differential inclusions driven by a fractional Brownian motion with infinite delay in the case where the right hand side is convex or nonconvex-valued. The results are obtained by using two fixed point theorems for multivalued mappings.
\end{abstract}

\section{INTRODUCTION}

The theory of stochastic differential and partial differential equations or inclusions has become an active area of investigation due to their applications in the fields such as mechanics, electrical engineering, medicine biology, ecology etc.

In recent years, stochastic differential and partial differential inclusions have been extensively studied. For instance, in $[1,2,3,5]$ it is investigated the existence of solutions of nonlinear stochastic differential inclusions by Banach fixed point theorem and semigroup approach. Balasubramaniam [4] obtained existence of solutions of functional stochastic differential inclusions by Kakutani's fixed point theorem, Balasubramaniam et al. $[5,6]$ initiated the study of existence of solutions of semilinear stochastic delay evolution inclusions in a Hilbert space by using the nonlinear alternative of Leray-Schauder type [15]. In [25] the authors study the existence results for impulsive neutral stochastic evolution inclusions in Hilbert spaces where they considered a class of first-order evolution inclusions with convex and nonconvex cases for the above problem by a fixed point theorem due to Dhage and Covitz and Nadler's theorem for contraction multivalued maps (see [16]).

Meanwhile, impulsive differential systems and evolution differential systems are used to describe various models of real processes and phenomena studied in physics, chemical technology, population dynamics, biotechnology and economics. That is why in recent years they have been the object of investigations. We refer to the monographs of Bainov and Simeonov [7], Benchohra et al. [8], Lakshmikantham et al. [22], Samoilenko and Perestyuk [28] where numerous properties of their solutions are studied, and a detailed bibliography is given. Semilinear functional differential equations and inclusions with or without impulses have been extensively studied where the operator $\mathrm{A}$ generates a $\mathrm{C}_{0}$-semigroup. Existence and uniqueness, among other things, are derived; see the books of Dejabli et al. [14] and Graef et al [18].

1991 Mathematics Subject Classification. 34A60,60H10,60H20.

Key words and phrases. mild solutions, impulsive stochastic functional differential inclusion, fractional Brownian motion, fixed point. 
The existence of neutral stochastic functional differential equations driven by a fractional Brownian motion has attracted great interest of researchers. For example, Boufoussi and Hajji analyzed in [12] the existence and uniqueness of mild solutions for a neutral stochastic differential equation with finite delay, driven by a fractional Brownian motion in a Hilbert space, and established some sufficient conditions ensuring the exponential decay to zero in mean square for the mild solution. Caraballo and Diop [13] studied the existence and uniqueness of mild solutions to neutral stochastic delay functional integro-differential equations perturbed by a fractional Brownian motion.

Recently, Boudaoui et al. [11] proved the existence of mild solutions to stochastic impulsive evolution equations with time delays, driven by fractional Brownian motion and Krasnoselski-Schaefer type fixed point theorem.

Let $B_{Q}^{H}$ is a fractional Brownian motion on a real and separable Hilbert space $\mathcal{K}$, with Hurst parameter $H \in(1 / 2,1)$, and with respect to a complete probability space $\left(\Omega, \mathcal{F}, \mathcal{F}_{t}, P\right)$ furnished with a family of right continuous and increasing $\sigma$ algebras $\left\{\mathcal{F}_{t}, t \in J\right\}$ satisfying $\mathcal{F}_{t} \subset \mathcal{F}$. We are interested in the existence problem of the following stochastic differential inclusions:

$$
\left\{\begin{array}{l}
d y(t) \in\left[A y(t)+F\left(t, y_{t}\right)\right] d t+g(t) d B_{Q}^{H}(t), \quad t \in J=[0, T], t \neq t_{k}, \\
y\left(t_{k}^{+}\right)-y\left(t_{k}^{-}\right)=I_{k}\left(y\left(t_{k}^{-}\right)\right), k=1, \ldots, m, \quad J_{0}=(-\infty, 0], \\
y(t)=\phi(t) \in \mathcal{D},
\end{array}\right.
$$

in a real separable Hilbert space $\mathcal{H}$ with inner product $(\cdot, \cdot)$ and norm $\|\cdot\|$, where $A$ : $D(A) \subset \mathcal{H} \longrightarrow \mathcal{H}$ is the infinitesimal generator of a strongly continuous semigroup of bounded linear operators $S(t), 0 \leq t \leq T$.

Assume $F: J \times \mathcal{D} \longrightarrow \mathcal{P}(\mathcal{H})$ is a bounded, closed and convex-valued multivalued map, $g: J \rightarrow L_{Q}^{0}(\mathcal{K}, \mathcal{H})$. Here, $L_{Q}^{0}(\mathcal{K}, \mathcal{H})$ denotes the space of all $Q$-Hilbert-Schmidt operators from $\mathcal{K}$ into $\mathcal{H}$, which will be also defined in the next section.

As for the impulse functions we will assume that $I_{k} \in C(\mathcal{H}, \mathcal{H})(k=1,2, \ldots, m)$ are bounded. Moreover, the fixed times $t_{k}$ satisfy $0<t_{1}<t_{2}<\ldots<t_{m}<T, y\left(t_{k}^{-}\right)$ and $y\left(t_{k}^{+}\right)$denotes the left and right limits of $y(t)$ at $t=t_{k}$. As for $y_{t}$ we mean the segment solution which is define in the usual way, that is, if $y(\cdot, \cdot):(-\infty, T] \times \Omega \rightarrow$ $\mathcal{H}$, then for any $t \geq 0, y_{t}(\cdot, \cdot):(-\infty, 0] \times \Omega \rightarrow \mathcal{H}$ is given by

$$
y_{t}(\theta, \omega)=y(t+\theta, \omega), \text { for } \theta \in(-\infty, 0], \omega \in \Omega,
$$

belong to some abstract phase space $\mathcal{D}$, that is a phase space defined axiomatically. Thus $\mathcal{D}$ is a linear space of functions mapping $[0, T] \times \Omega$ into $\mathcal{H}$ endowed with a seminorm $\|\cdot\|_{\mathcal{D}_{T}}$. Consider the following space

$$
\begin{array}{r}
\mathcal{D}_{T}:=\left\{y:[0, T] \rightarrow \mathcal{H}, \quad \text { such that }\left.y\right|_{J_{k}} \in C\left(J_{k}, \mathcal{H}\right) \text { and there exist } y\left(t_{k}^{+}\right),\right. \\
\text {and } \left.y\left(t_{k}^{-}\right) \text {with } y\left(t_{k}\right)=y\left(t_{k}^{-}\right), k=1, \cdots, m, \sup _{t \in[0, T]} E\left(|y(t)|^{2}\right)<\infty\right\}
\end{array}
$$

where $\left.y\right|_{J_{k}}$ is the restriction of $y$ to $J_{k}=\left(t_{k}, t_{k+1}\right], k=1,2, \cdots, m$. We endow $\mathcal{D}_{T}$ with a norm $\|\cdot\|_{\mathcal{D}_{T}}$ on $\mathcal{D}_{T}$ defined by

$$
\|y\|_{\mathcal{D}_{T}}=\sup _{0 \leq s \leq T}\left(E\|y(s)\|^{2}\right)^{\frac{1}{2}}
$$

We will assume that $\mathcal{D}$ satisfies the following axioms suggested by Hale and Kato [19] and Hino et al. [20]. The axioms of the space $\mathcal{D}$ are established for 
$\mathcal{F}_{0}$-measurable functions from $J_{0}$ into $\mathcal{H}$, endowed with the seminorm $\|\cdot\|_{\mathcal{D}}$. We will assume that $\mathcal{D}$ satisfies the following axioms:

(A-1): If $y:(-\infty, T] \longrightarrow \mathcal{H}, T>0$ is such that $\left.y\right|_{[0, T]} \in \mathcal{D}$ and $y_{0} \in \mathcal{D}$, then for every $t \in[0, T)$ the following conditions hold

(i): $y_{t}$ is in $\mathcal{D}$

(ii): $\|y(t)\| \leq L\left\|y_{t}\right\|_{\mathcal{D}}$

(iii): $\left\|y_{t}\right\|_{\mathcal{D}} \leq K(t) \sup \|y(s)\|: 0 \leq s \leq t+N(t)\left\|y_{0}\right\|_{\mathcal{D}}$, where $L>0$ is a constant; $K, N:[0, \infty) \longrightarrow[0, \infty), K$ is continuous, $N$ is locally bounded and $L, K, N$ are independent of $y(\cdot)$.

(A-2): For the function $y(\cdot)$ in $(\mathrm{A}-1), y_{t}$ is a $\mathcal{D}$-valued function $[0, T)$.

(A-3): The space $\mathcal{D}$ is complete.

Set

$$
\mathcal{D}_{T}^{*}=\left\{y:(-\infty, T] \longrightarrow \mathcal{H}, y \in \mathcal{D}_{T} \cap \mathcal{D}\right\}
$$

$\|\cdot\|_{\mathcal{D}_{T}^{*}}$ on $\mathcal{D}_{T}^{*}$, it is defined by

$$
\|y\|_{\mathcal{D}_{T}^{*}}=\sup _{0 \leq s \leq T}\left(E\|y(s)\|^{2}\right)^{\frac{1}{2}}=\|\phi\|_{\mathcal{D}}+\sup _{0 \leq s \leq T}\left(E\|y(s)\|^{2}\right)^{\frac{1}{2}}, y \in \mathcal{D}_{T}^{*} .
$$

Denote

$$
\widetilde{K}=\sup \{K(t): t \in J\} \text { and } \widetilde{M}=\sup \{M(t): t \in J\} .
$$

The plan of this paper is as follows. In Section 2 we introduce notations, definitions, and preliminary facts which are useful throughout the paper. In Section 3 we prove existence of mild solutions for problem (1.1), where the right-hand side is convex or nonconvex by some appropriate fixed point theorems.

\section{Preliminaries}

In this section, we introduce notations, definitions, and preliminary facts which will be used throughout this paper.

In particular, we consider fractional Brownian motion as well as the Wiener integral with respect to it. We also establish some important results which will be needed throughout the paper.

Definition 2.1. Given $H \in(0,1)$, a continuous centered Gaussian process $\beta^{H}=$ $\left\{\beta^{H}(t), t \in \mathbb{R}\right\}$, with the covariance function

$$
R_{H}(t, s)=E\left[\beta^{H}(t) \beta^{H}(s)\right]=\frac{1}{2}\left(|t|^{2 H}+|s|^{2 H}-|t-s|^{2 H}\right), t, s \in \mathbb{R}
$$

is called a two-sided one-dimensional fractional Brownian motion, and $H$ is the Hurst parameter.

Now we aim at introducing the Wiener integral with respect to the one-dimensional $\beta^{H}$.

Let $T>0$ and denote by $\Lambda$ the linear space of $\mathbb{R}$-valued step functions on $[0, T]$, that is, $\psi \in \Lambda$ if

$$
\psi(t)=\sum_{i=1}^{n-1} y_{i} 1_{\left[s_{i}, s_{i-1}\right)}(t),
$$


where $t \in[0, T], y_{i} \in \mathbb{R}$ and $0=s_{1}<s_{2}<\cdots<s_{n}=T$. For $\psi \in \Lambda$ we define its Wiener integral with respect to $\beta^{H}$ as

$$
\int_{0}^{T} \psi(\sigma) d \beta^{H}(\sigma)=\sum_{i=1}^{n-1} y_{i}\left(\beta^{H}\left(s_{i+1}\right)-\beta^{H}\left(s_{i}\right)\right) .
$$

Let $\mathcal{H}$ be the Hilbert space defined as the closure of $\Lambda$ with respect to the scalar product

Then, the mapping

$$
\left\langle 1_{[0, t]}, 1_{[0, s]}\right\rangle_{\mathcal{H}}=R_{H}(t, s) .
$$

$$
\psi=\sum_{i=1}^{n-1} y_{i} 1_{\left[s_{i}, s_{i+1}\right)} \mapsto \int_{0}^{T} \psi(\sigma) d \beta^{H}(\sigma)
$$

is an isometry between $\Lambda$ and the linear space span $\left\{\beta^{H}(t), t \in[0, T]\right\}$, which can be extended to an isometry between $\mathcal{H}$ and the first Wiener chaos of the fractional Brownian motion $\overline{\operatorname{span}}^{L^{2}(\Omega)}\left\{\beta^{H}(t), t \in[0, T]\right\}$ (see [27]). The image of an element $\psi \in \mathcal{H}$ by this isometry is called the Wiener integral of $\psi$ with respect to $\beta^{H}$. Our next goal is to give an explicit expression of this integral. To this end, consider the kernel

$$
K_{H}(t, s)=c_{H} s^{1 / 2-H} \int_{s}^{t}(u-s)^{H-3 / 2} u^{H-1 / 2} d u,
$$

where $c_{H}=\left(\frac{H(2 H-1)}{B\left(2-2 H, H-\frac{1}{2}\right)}\right)^{1 / 2}$, with $B(\cdot, \cdot)$ denoting the Beta function, and $t \leq s$.

It is not difficult to see that

$$
\frac{\partial K_{H}}{\partial t}(t, s)=c_{H}\left(\frac{t}{s}\right)^{\frac{1}{2}-H}(t-s)^{H-\frac{3}{2}} .
$$

Consider the linear operator $K_{H}^{*}: \Lambda \longrightarrow L^{2}([0, T]$ given by

Then

$$
\left(K_{H}^{*} \Phi\right)(s)=\int_{s}^{t} \Phi(t) \frac{\partial K_{H}}{\partial t}(t, s) d t .
$$

$$
\left(K_{H}^{*} 1_{[0, t]}\right)(s)=K_{H}(t, s) 1_{[0, t]}(s) .
$$

and $K_{H}^{*}$ is an isometry between $\Lambda$ and $L^{2}([0, T])$ that can be extended to $\Lambda$ (see [3]). Considering $W=\{W(t), t \in[0, T]\}$ defined by

$$
W(t)=\beta^{H}\left(\left(K_{H}^{*}\right)^{-1} 1_{[0, t]}\right),
$$

it turns out that $W$ is a Wiener process and $\beta^{H}$ has the following Wiener integral representation:

In addition, for any $\Phi \in \Lambda$,

$$
\beta^{H}(t)=\int_{0}^{t} K_{H}(t, s) d W(s) .
$$

$$
\int_{0}^{T} \Phi(s) \beta^{H}(s) d W(s)=\int_{0}^{T}\left(K_{H}^{*} \Phi\right)(t) d W(t)
$$

if and only if $K_{H}^{*} \Phi \in L^{2}([0, T])$.

Also denoting

$$
L_{\mathcal{H}}^{2}([0, T])=\left\{\Phi \in \Lambda, K_{H}^{*} \Phi \in L^{2}([0, T])\right\}
$$


since $H>1 / 2$, we have

$$
L^{1 / H}([0, T]) \subset L_{\mathcal{H}}^{2}([0, T]),
$$

see [24]. Moreover, the following useful result holds:

Lemma 2.2. [26]. For $\Phi \in L^{1 / H}([0, T])$,

$$
H(2 H-1) \int_{0}^{T} \int_{0}^{T}|\Phi(r)\|\Phi(u)\| r-u|^{2 H-2} d r d u \leq c_{H}\|\Phi\|_{L^{1 / H}([0, T])}^{2} .
$$

Next we are interested in considering a fractional Brownian motion with values in a Hilbert space and giving the definition of the corresponding stochastic integral.

Let $L(\mathcal{K}, \mathcal{H})$ denote the space of all bounded linear operators from $\mathcal{K}$ to $\mathcal{H}$ and $Q \in L(\mathcal{K}, \mathcal{H})$ be a non-negative self-adjoint operator. Denote by $L_{Q}^{0}(\mathcal{K}, \mathcal{H})$ the space of all $\xi \in L(\mathcal{K}, \mathcal{H})$ such that $\xi Q^{\frac{1}{2}}$ is a Hilbert-Schmidt operator. The norm is given by

$$
|\xi|_{L_{Q}^{0}(\mathcal{K}, \mathcal{H})}^{2}=\operatorname{tr}\left(\xi Q \xi^{*}\right) .
$$

Then $\xi$ is called a $Q$-Hilbert-Schmidt operator from $\mathcal{K}$ to $\mathcal{H}$.

Let $\left\{\beta_{n}^{H}(t)\right\}_{n \in N}$ be a sequence of two-sided one-dimensional standard fractional Brownian motions mutually independent on $(\Omega, \mathcal{F}, P)$. When one considers the following series

$$
\sum_{n=1}^{\infty} \beta_{n}^{H}(t) e_{n}, \quad t \geq 0
$$

where $\left\{e_{n}\right\}_{n \in N}$ is a complete orthonormal basis in $\mathcal{K}$, this series does not necessarily converge in the space $\mathcal{K}$. Thus we consider a $\mathcal{K}$-valued stochastic process $B_{Q}^{H}(t)$ given formally by the following series:

$$
B_{Q}^{H}(t)=\sum_{n=1}^{\infty} \beta_{n}^{H}(t) Q^{\frac{1}{2}} e_{n}, \quad t \geq 0
$$

is well-defined as a $\mathbb{K}$-valued $Q$-cylindrical fractional Brownian motion.

Let $\varphi:[0, T] \mapsto L_{0}^{Q}(\mathcal{K}, \mathcal{H})$ such that

$$
\sum_{n=1}^{\infty}\left\|K_{H}^{*}\left(\varphi Q^{\frac{1}{2}} e_{n}\right)\right\|_{L^{1 / H}([0, T], \mathcal{H})}<\infty
$$

Definition 2.3. Let $\varphi:[0, T] \longrightarrow L_{Q}^{0}(\mathcal{K}, \mathcal{H})$ satisfy (2.2). Then, its stochastic integral with respect to the fractional Brownian motion $B_{Q}^{H}$ is defined, for $t \geq 0$, as follows

$$
\int_{0}^{t} \varphi(s) d B_{Q}^{H}(s):=\sum_{n=1}^{\infty} \int_{0}^{t} \varphi(s) Q^{1 / 2} e_{n} d \beta_{n}^{H}(s)=\sum_{n=1}^{\infty} \int_{0}^{t}\left(K_{H}^{*}\left(\varphi Q^{1 / 2} e_{n}\right)\right)(s) d W(s) .
$$

Notice that if

$$
\sum_{n=1}^{\infty}\left\|\varphi Q^{1 / 2} e_{n}\right\|_{L^{1 / H}([0, T] ; \mathcal{H})}<\infty
$$

then in particular (2.2) holds, which follows immediately from (2.1). 
Lemma 2.4. [12] if $\varphi:[0, T] \longrightarrow L_{Q}^{0}(\mathcal{K}, \mathcal{H})$ satisfies

$$
\int_{0}^{T}\|\varphi(s)\|_{L_{Q}^{0}(\mathcal{K}, \mathcal{H})}^{2} d s<\infty
$$

and for any $\alpha, \beta \in[0, T]$ with $\alpha>\beta$, then the above sum in (2.3) is well defined as a $\mathcal{H}$-valued random variable and we have

$$
E\left|\int_{\beta}^{\alpha} \varphi(s) d B_{Q}^{H}(s)\right|^{2} \leq 2 H(\alpha-\beta)^{2 H-1} \int_{\beta}^{\alpha}\|\varphi(s)\|_{L_{Q}^{0}(\mathcal{K}, \mathcal{H})}^{2} d s .
$$

Let us introduce the following notations:

$$
\begin{gathered}
\mathcal{P}_{c l}(\mathcal{H})=\{A \in \mathcal{P}(\mathcal{H}): A \text { closed }\}, \\
\mathcal{P}_{b}(\mathcal{H})=\{A \in \mathcal{P}(\mathcal{H}): A \text { bounded }\}, \\
\mathcal{P}_{c}(\mathcal{H})=\{A \in \mathcal{P}(\mathcal{H}): A \text { convex }\}, \\
\mathcal{P}_{c p}(\mathcal{H})=\{A \in \mathcal{P}(\mathcal{H}): A \text { compact }\}, \\
\mathcal{P}_{c p, c}(\mathcal{H})=\{A \in \mathcal{P}(\mathcal{H}): A \text { compact and convex }\}, \\
\mathcal{P}_{b, c l}(\mathcal{H})=\{A \in \mathcal{P}(\mathcal{H}): A \text { bounded and closed }\} .
\end{gathered}
$$

Consider $H_{d}: \mathcal{P}(\mathcal{H}) \times \mathcal{P}(\mathcal{H}) \longrightarrow \mathbb{R} \cup\{\infty\}$ given by

$$
H_{d}(A, B)=\max \left\{\sup _{a \in A} d(a, B), \sup _{b \in B} d(A, b)\right\},
$$

where $d(A, b)=\inf _{a \in A} d(a, b), d(a, B)=\inf _{b \in B} d(a, b)$. Then, $\left(\mathcal{P}_{b, c l}(\mathcal{H}), H_{d}\right)$ is a metric space and $\left(\mathcal{P}_{c l}(\mathcal{H}), H_{d}\right)$ is a generalized metric space [21].

A multivalued map $F: \mathcal{H} \longrightarrow \mathcal{P}(\mathcal{H})$ is convex (closed) valued if $F(y)$ is convex (closed) for all $y \in \mathcal{H}, F$ is bounded on bounded sets if $F(B)=\bigcup_{y \in B} F(y)$ is bounded in $\mathcal{H}$ for all $B \in \mathcal{P}_{b}(\mathcal{H})$, i.e., $\sup _{x \in B}\{\sup \{|y|: y \in F(y)\}\} \leq \infty$. F is called upper semi-continuous (u.s.c. for short) on $\mathcal{H}$ if for each $y_{0} \in \mathcal{H}$ the set $F\left(y_{0}\right)$ is a nonempty of $\mathcal{H}$, and for each open set $\mathcal{U}$ of $\mathcal{H}$ containing $F\left(y_{0}\right)$, there exists an open neighborhood $\mathcal{V}$ of $y_{0}$ such that $F(\mathcal{V}) \in \mathcal{U} . F$ is said to be completely continuous if $F(B)$ is relatively compact for every $B \in \mathcal{P}_{b}(\mathcal{H})$.

If the multivalued map $F$ is completely continuous with nonempty compact valued, then $F$ is u.s.c. if and only if $F$ has a closed graph, i.e., $x_{n} \longrightarrow x_{*}, y_{n} \longrightarrow y_{*}$, $y_{n} \in F\left(x_{n}\right)$ imply $y_{*} \in F\left(x_{*}\right)$.

$A$ multi-valued map $F: J \longrightarrow \mathcal{P}_{c l}(H)$ is said to be measurable if for each $y \in \mathcal{H}$, the mean-square distance between $y$ and $F(t)$ is measurable.

Definition 2.5. The multi-valued map $F: J \times \mathcal{D} \rightarrow \mathcal{P}(\mathcal{H})$ is said to be $L^{2}$ Carathéodory if

(i): $t \mapsto F(t, v)$ is measurable for each $v \in \mathcal{H}$;

(ii): $v \mapsto F(t, v)$ is u.s.c. for almost all $t \in J$;

(iii): for each $q>0$, there exists $h_{q} \in L^{1}\left(J, \mathbb{R}^{+}\right)$such that

$$
\|F(t, v)\|^{2}:=\sup _{f \in F(t, v)} E\|f\|^{2} \leq h_{q}(t) \text {, for all }\|v\|_{\mathcal{D}}^{2} \leq q \text { and for a.e. } t \in J .
$$

Lemma 2.6. [23] Let $I$ be a compact interval and $\mathcal{H}$ be a Hilbert space. Let $F$ be an $L^{2}$-Carathéodory multi-valued map with $N_{F, y} \neq \emptyset$. and let $\Gamma$ be a linear continuous mapping from $L^{2}(I, \mathcal{H})$ to $C(I, \mathcal{H})$. Then, the operator

$$
\Gamma \circ N_{F}: C(I, \mathcal{H}) \longrightarrow \mathcal{P}_{c p, c}(\mathcal{H}), y \longmapsto\left(\Gamma \circ N_{F}\right)(y)=\Gamma\left(N_{F}, y\right),
$$


is a closed graph operator in $C(I, \mathcal{H}) \times C(I, \mathcal{H})$, where $N_{F, y}$ is known as the selectors set from $F$ and given by

$$
f \in N_{F, y}=\left\{f \in L^{2}([0, T], \mathcal{H}): f(t) \in F(t, y) \text { for a.e.t } \in[0, T]\right\} \text {. }
$$

Definition 2.7. A multivalued operator $G: J \longrightarrow \mathcal{P}_{c l}(\mathcal{H})$ is called a contraction if and only if there exists $0 \leq \gamma<1$ such that

$$
H_{d}(G(x), G(y)) \leq \gamma d(x, y) \text { for each } x, y \in \mathcal{H},
$$

and we say that a multivalued operator $G$ has a fixed point if there exists $y \in \mathcal{H}$ such that $y \in G(y)$.

Now we recall a Leray-Schauder a fixed point theorem which will be useful for our analysis

Lemma 2.8. [16] Let $X$ be a Banach space with $C \subset X$ a closed and convex subset of $X$. Assume $U$ is a relatively open subset of $C$, with $0 \in U$, and let $G: \bar{U} \longrightarrow C$ be a compact map. Then either,

(a): G has a fixed point in $\bar{U}$, or

(b): there is a point $u \in \partial U$ and $\lambda \in(0,1)$, with $u=\partial G(u)$.

\section{Existence RESUlts}

3.1. The Convex Case. In this section, we will show same results concerning the existence results of mild solutions for convex case of system (1.1)in the convex case. Our main results are based on the following lemma.

Lemma 3.1. $[15]$

Let $X$ be a Banach space with $C \subset X$ convex. Assume $U$ is a relatively open subset of $C$, with $0 \in U$, and let $G: X \longrightarrow \mathcal{P}_{c p, c}(X)$ be an upper semicontinuous and compact map. Then either,

(a): $G$ has a fixed point in $\bar{U}$, or

(b): there is a point $u \in \partial U$ and $\lambda \in(0,1)$, with $u \in \partial G(u)$.

First, we define what we mean by a mild solution.

Definition 3.2. A stochastic process $y:(-\infty, T] \times \Omega \longrightarrow \mathcal{H}$ is called a mild solution of the system (1.1) if

- $y(t)$ is measurable and $\mathcal{F}_{t}$-adapted, for each $t \geq 0$;

- $y(t) \in \mathcal{H}$ has càdlàg paths on $t \in[0, T]$ a.s., for every $0 \leq s<t \leq T$, there exist $f \in N_{F, y}$ such that the following integral equation holds

$$
\begin{aligned}
y(t)= & S(t) \phi(0)+\int_{0}^{t} S(t-s) f(s) d s+\int_{0}^{t} S(t-s) g(s) d B_{Q}^{H}(s) \\
& +\sum_{0<t_{k}<t} S\left(t-t_{k}\right) I_{k}\left(y\left(t_{k}^{-}\right)\right), t \in J .
\end{aligned}
$$

- $y_{0}(\cdot)=\phi \in \mathcal{D}$ on $J_{0}$ satisfies $\|\phi\|_{\mathcal{D}}<\infty$.

We are now in a position to state and prove our existence result for the problem (1.1). First we will list the following hypotheses which will be imposed in our main theorem.

(H1): Operator $A: D(A) \subset \mathcal{H} \rightarrow \mathcal{H}$ is the infinitesimal generator of a strongly continuous semigroup of bounded linear operators $\{S(t)\}, t \in J$ which is compact for $t>0$ in $\mathcal{H}$ such that $\|S(t)\|^{2} \leq M$ for same $M \geq 0$ and for each $t \in[0, T]$. 
(H2): The function $g: J \longrightarrow L_{Q}^{0}(\mathcal{K}, \mathcal{H})$ satisfies

$$
\int_{0}^{T}\|g(s)\|_{L_{Q}^{0}}^{2} d s=\Lambda<\infty, t \in J
$$

(H3): There exist constants $c_{k}>0, k=1, \ldots, m$ for which $\left|I_{k}(y)\right|^{2} \leq$ $c_{k}$, for all $y \in \mathcal{H}$.

(H4): $F:[0, T] \times \mathcal{D} \longrightarrow \mathcal{P}_{c p, c}(\mathcal{H})$ is an $L^{2}$-Carathdory function and for every $t \in[0, T]$ the multifunction $t \rightarrow F\left(t, y_{t}\right), y_{t} \in \mathcal{D}$ is measurable.

(H5): There exist $\eta \in L^{2}\left(J, \mathbb{R}^{+}\right)$and $p: \mathbb{R}^{+} \longrightarrow(0, \infty)$ is continuous and increasing such that

$$
\left.E|F(t, \Theta)|^{2}=\left\{\sup E(|f|)^{2}: f \in F(t, \Theta)\right\}\right\} \leq \eta(t) p\left(\|\Theta\|_{\mathcal{D}}^{2}\right), \quad t \in J, \quad \Theta \in \mathcal{D},
$$

where

$$
8 \widetilde{K}^{2} M\left(t_{k}-t_{k-1}\right) \int_{t_{k-1}}^{t_{k}} \eta(s) d s \leq \int_{N_{k-1}}^{\infty} \frac{d u}{p(u)}, k=1, \cdots, m+1,
$$

and

$$
\begin{aligned}
& N_{0}=v_{0}(0)=16 \widetilde{K}^{2} M H t_{1}^{2 H-1} \int_{0}^{t_{1}}\|g(s)\|_{L_{Q}^{0}}^{2} d s+C \\
& C=4 \widetilde{K}^{2} M E|\widehat{\phi}(0)|^{2}+4 \widetilde{M}^{2}\|\widehat{\phi}\|_{\mathcal{D}}^{2} \\
& \text { and for } k=2, \cdots, m+2 \\
& N_{k-1}=4 \widetilde{K}^{2}\left[4 M \sup _{y \in\left(-\infty, t_{k-1}\right]} E\left|I_{k-1}\left(y_{t_{2-1}}\left(t_{k-1}\right)\right)\right|^{2}\right. \\
& \left.\quad+8 \widetilde{K}^{2} M\left(t_{k-1}-t_{k-2}\right)^{2 H-1} \int_{t_{k-2}}^{t_{k-1}}\|g(s)\|_{L_{Q}^{0}}^{2} d s\right]+C \\
& \widehat{M}_{k-2}=\Gamma_{k-1}^{-1}\left(8 \widetilde{K}^{2} M\left(t_{k-1}-t_{k-2}\right) \int_{t_{k-2}}^{t_{k-1}} \eta(s) d s\right)
\end{aligned}
$$

with

$$
\Gamma_{l}(x)=\int_{N_{l-1}}^{x} \frac{d u}{p(u)} x \geq N_{l-1}, l \in\{1, \cdots, m+1\} .
$$

Theorem 3.3. Assume that hypotheses $(H 1)-(H 5)$ hold. Then the problem (1.1) has at least one integral solution on $(-\infty, T]$.

Proof. The proof will be given in several steps.

Step1. Consider the problem (1.1) on $\left(-\infty, t_{1}\right]$

$$
\begin{array}{ll}
d y(t) \in\left[A y(t)+F\left(t, y_{t}\right)\right] d t+g(t) d B_{Q}^{H}(t), & \text { if } t \in\left[0, t_{1}\right], \\
y(t)=\phi(t), & \text { if } t \in(-\infty, 0] .
\end{array}
$$

Let

$$
\mathcal{D}_{t_{0}}=\left\{y \in C\left(\left[0, t_{1}\right], \mathcal{H}\right): \sup _{t \in\left[0, t_{1}\right]} E\left(|y(t)|^{2}\right)<\infty\right\}
$$

Set

$$
\mathcal{D}_{t_{0}}^{*}=\mathcal{D} \cap \mathcal{D}_{t_{0}}
$$


We transform the problem (3.2) into a fixed point problem. Consider the multivalued operator $\Phi: \mathcal{D}_{t_{0}}^{*} \rightarrow \mathcal{P}\left(\mathcal{D}_{t_{0}}^{*}\right)$ defined by

$$
\Phi(y)=\left\{\rho \in \mathcal{D}_{t_{1}} \rho(t)=\left\{\begin{array}{ll}
\phi(t), & \text { if } ; \in(-\infty, 0], \\
S(t) \phi(0)+\int_{0}^{t} S(t-s) f(s) d s & \\
+\int_{0}^{t} S(t-s) g(s) d B^{H}(s), & \text { if } t \in\left[0, t_{1}\right]
\end{array}\right\}\right.
$$

where $f \in N_{F, y}=\left\{f \in L^{2}\left(\left[0, t_{1}\right], \mathcal{H}\right): f(t) \in F\left(t, y_{t}\right)\right.$ for a.e.t $\left.\in\left[0, t_{1}\right]\right\}$.

We will prove that $\Phi$ has a fixed point.

Let $\widehat{\phi}:\left(-\infty, t_{1}\right] \longrightarrow \mathcal{H}$ be the function defined by

$$
\widehat{\phi}(t)= \begin{cases}\phi(t), & t \in(-\infty, 0] \\ S(t) \phi(0), & t \in\left[0, t_{1}\right]\end{cases}
$$

Then $\widehat{\phi}$ is an element of $\mathcal{D}_{t_{0}}^{*}$ and $\widehat{\phi}_{0}=\phi$.

Let $y(t)=z(t)+\widehat{\phi}(t),-\infty<t \leq t_{1}$. Obviously, if $y$ satisfies the integral equation

$$
y(t)=S(t) \phi(0)+\int_{0}^{t} S(t-s) f(s) d s+\int_{0}^{t} S(t-s) g(s) d B_{Q}^{H}(s), t \in\left[0, t_{1}\right] .
$$

then $z$ satisfies $z_{0}=0, t \in(-\infty, 0]$ and

$$
z(t)=\int_{0}^{t} S(t-s) f(s) d s+\int_{0}^{t} S(t-s) g(s) d B_{Q}^{H}(s), t \in\left[0, t_{1}\right]
$$

where $f(t) \in F\left(t, z_{t}+\widehat{\phi}_{t}\right)$ for a.e. $t \in\left[0, t_{1}\right]$.

Set $\mathcal{D}_{t_{0}}^{0}=\left\{z \in \mathcal{D}_{t_{0}}^{*}\right.$, such that $\left.z_{0}=0 \in \mathcal{D}\right\}$ and for any $z \in \mathcal{D}_{t_{0}}^{0}$ we have

$$
\|y\|_{\mathcal{D}_{t_{0}}^{0}}=\left\|z_{0}\right\|_{\mathcal{D}}+\sup _{t \in\left[0, t_{1}\right]}\left(E\|z(t)\|^{2}\right)^{\frac{1}{2}}=\sup _{t \in\left[0, t_{1}\right]}\left(E\|y(t)\|^{2}\right)^{\frac{1}{2}}
$$

Thus $\left(\mathcal{D}_{t_{0}}^{0},\|\cdot\|_{\mathcal{D}_{t_{0}}^{0}}\right)$ is a Banach space. Let the operator $\widehat{\Phi}: \mathcal{D}_{t_{0}}^{0} \rightarrow \mathcal{P}\left(\mathcal{D}_{t_{0}}^{0}\right)$ defined by

$$
\widehat{\Phi}(z)=\left\{\widehat{\rho} \in \mathcal{D}_{t_{0}}^{0} \widehat{\rho}(t)=\left\{\begin{array}{ll}
0, & \text { if; } t \in(-\infty, 0], \\
\int_{0}^{t} S(t-s) f(s) d s+\int_{0}^{t} S(t-s) g(s) d B^{H}(s) & \text { if } t \in\left[0, t_{1}\right] .
\end{array}\right\}\right.
$$

Clearly, that the operator $\Phi$ has a fixed point is equivalent to $\widehat{\Phi}$ having a fixed point, and so we turn our attention to proving that $\widehat{\Phi}$ does in fact have a fixed point. We shall show that $\widehat{\Phi}$ satisfies the assumptions of Lemma 3.1.

Claim 1. $\widehat{\Phi}(z)$ is convex for each $z \in \mathcal{D}_{t_{0}}^{0}$.

Let $\widehat{\rho}_{1}, \widehat{\rho}_{2} \in \widehat{\Phi}_{2}(z)$, then there exist $f_{1}, f_{2} \in N_{F, z+\widehat{\phi}}$ such that, for each $t \in\left[0, t_{1}\right]$ we have

$$
\widehat{\rho}_{i}(t)=\int_{0}^{t} S(t-s) f_{i}(s) d s+\int_{0}^{t} S(t-s) g(s) d B^{H}(s) .
$$

Let $0 \leq \delta \leq 1$. Then, for each $t \in\left[0, t_{1}\right]$, we have

$$
\left(\delta \widehat{\rho}_{1}+(1-\delta) \widehat{\rho}_{2}\right)(t)=\int_{0}^{t} S(t-s)\left[\delta f_{1}(s)+(1-\delta) f_{2}(s)\right] d s+\int_{0}^{t} S(t-s) g(s) d B^{H}(s)
$$


Since $N_{F, z+\widehat{\phi}}$ is convex $(F(t, z)$ has convex values), one has

$$
\delta \widehat{\rho}_{1}+(1-\delta) \widehat{\rho}_{2} \in \widehat{\Phi}(z)
$$

Claim 2. $\widehat{\Phi}$ maps bounded sets into bounded sets in $\mathcal{D}_{t_{0}}^{0}$.

Indeed, it is enough to show that there exists a positive constant $\zeta$ such that for each $\widehat{\rho} \in \widehat{\Phi}_{z}, w \in \mathcal{B}_{q}=\left\{z \in \mathcal{D}_{t_{0}}^{0},\|z\|_{\mathcal{D}_{t_{0}}^{0}}^{2} \leq q\right\}$ one has $\|\widehat{\rho}\|_{\mathcal{D}_{t_{0}}^{0}}^{2} \leq \zeta$.

Let $\widehat{\rho} \in \widehat{\Phi}$, then there exists $f \in N_{F, z+\widehat{\phi}}$ such that for $t \in\left[0, t_{1}\right]$, we have

$$
\widehat{\rho}(t)=\int_{0}^{t} S(t-s) f(s) d s+\int_{0}^{t} S(t-s) g(s) d B^{H}(s) .
$$

From (A1) we have

$$
\begin{aligned}
\left\|z_{t}+\widehat{\phi}_{t}\right\|_{\mathcal{D}}^{2} \leq & 2\left(\left\|z_{t}\right\|_{\mathcal{D}}^{2}+\left\|\widehat{\phi}_{t}\right\|_{\mathcal{D}}^{2}\right) \\
& \leq 4\left((k(t))^{2} \sup _{s \in[0, t]} E|z(s)|^{2}+(M(t))^{2}\left\|z_{0}\right\|_{\mathcal{D}}^{2}\right) \\
& +4\left((K(t))^{2} \sup _{s \in[0, t]} E\|\widehat{\phi}(s)\|^{2}+(M(t))^{2}\left\|\widehat{\phi}_{0}\right\|_{\mathcal{D}}^{2}\right) \\
& \leq 4\left(\widetilde{M}^{2}\|\phi\|_{\mathcal{D}}^{2}+\widetilde{K}^{2}\left(q+M E|\phi(0)|^{2}\right)\right)=q^{\prime} .
\end{aligned}
$$

From $(H 1)-(H 4)$, we obtain for $t \in\left[0, t_{1}\right]$,

$$
\begin{aligned}
E|\widehat{\rho}(t)|^{2} & =E\left|\int_{0}^{t} S(t-s) f(s) d s+\int_{0}^{t} S(t-s) g(s) d B^{H}(s)\right|^{2} \\
& \leq 2 E\left|\int_{0}^{t} S(t-s) f(s) d s\right|^{2}+2 E\left|\int_{0}^{t} S(t-s) g(s) d B^{H}(s)\right|^{2} \\
& \leq 2 M t_{1} \int_{0}^{t} E|f(s)|^{2} d s+4 M H t_{1}^{2 H-1} \int_{0}^{t_{1}}\|g(s)\|_{L_{Q}^{0}}^{2} d s \\
& \leq 2 M t_{1} p\left(q^{\prime}\right) \int_{0}^{t} \eta(s) d s+4 M H t_{1}^{2 H-1} \Lambda \\
& :=\zeta,
\end{aligned}
$$

Claim 3. $\widehat{\Phi}$ maps bounded sets into equicontinuous sets of $\mathcal{D}_{t_{0}}^{0}$.

Let $0<\tau_{1}<\tau_{2} \in\left[0, t_{1}\right], \mathcal{B}_{q}$ be a bounded set of $\mathcal{D}_{t_{0}}^{0}$ as in Claim 2. For each $z \in \mathcal{B}_{q}$ and $\widehat{\rho} \in \widehat{\Phi} z$, there exists $f \in N_{F, z+\widehat{\phi}}$ such that

$$
\widehat{\rho}(t)=\int_{0}^{t} S(t-s) f(s) d s+\int_{0}^{t} S(t-s) g(s) d B^{H}(s), \quad t \in\left[0, t_{1}\right] .
$$

Then we have

$$
\begin{aligned}
E\left|\widehat{\rho}\left(\tau_{2}\right)-\widehat{\rho}\left(\tau_{1}\right)\right|^{2} \leq & 4 t_{1} p\left(q^{\prime}\right) \int_{0}^{\tau_{2}}\left|S\left(\tau_{2}-s\right)-S\left(\tau_{1}-s\right)\right|^{2} \eta(s) d s \\
& +4 t_{1} p\left(q^{\prime}\right) \int_{\tau_{1}}^{\tau_{2}}\left|S\left(\tau_{1}-s\right)\right|^{2} \eta(s) d s \\
& +4 \int_{0}^{\tau_{2}}\left|S\left(\tau_{2}-s\right)-S\left(\tau_{1}-s\right)\right|^{2} E\left|g(s) d B^{H}(s)\right|^{2} \\
& +4 \int_{\tau_{1}}^{\tau_{2}}\left|S\left(\tau_{1}-s\right)\right|^{2} E\left|g(s) d B^{H}(s)\right|^{2}
\end{aligned}
$$

The right-hand side of the above inequality tends to zero as $\tau_{2} \longrightarrow \tau_{1}$, since $S(t)$ is strongly continuous operator and the compactness of $S(t)$ for $t>0$ implies 
the continuity in the uniform operator topology [29]. Thus, the set $\left\{\widehat{\Phi} z: z \in \mathcal{B}_{q}\right\}$ is equicontinuous.

As a consequence of Claims 2 and 3, together with the Arzelá-Ascoli theorem, it suffices to show that $\widehat{\Phi}$ maps $\mathcal{B}_{q}$ into a precompact set in $\mathcal{H}$.

Let $0<t<t_{1}$ be fixed and let $\epsilon$ be a real number satisfying $0<\epsilon<t$. For $z \in \mathcal{B}_{q}$ we define

$$
\left(\widehat{\Phi}_{\epsilon} z\right)(t)=S(\epsilon) \int_{0}^{t-\epsilon} S(t-s-\epsilon) f(s) d s+S(\epsilon) \int_{0}^{t-\epsilon} S(t-s-\epsilon) g(s) d B^{H}(s),
$$

where $f \in N_{F, z+\widehat{\phi}}$.

Since $S(t)$ is a compact operator, the set

$$
V_{\epsilon}(t)=\left\{\widehat{\Phi}_{\epsilon}(z)(t): z \in \mathcal{B}_{q}\right\}
$$

is precompact in $\mathcal{H}$ for every $\epsilon, 0<\epsilon<t$. Moreover, for every $z \in \mathcal{B}_{q}$ we have

$$
\begin{aligned}
E \mid(\widehat{\Phi} z)(t)-\left(\left.\widehat{\Phi}_{\epsilon}(z)(t)\right|^{2}\right. & \leq 2 t_{1} \int_{t-\epsilon}^{t}|S(t, s)|^{2} \eta(s) p(q) d s+2 \int_{t-\epsilon}^{t} S(t-\epsilon) E\left|g(s) d B^{H}(s)\right|^{2} \\
& \leq 2 t_{1} M \int_{t-\epsilon}^{t} \eta(s) p\left(q^{\prime}\right) d s+4 M H t_{1}^{2 H-1} \int_{0}^{t_{1}}\|g(s)\|_{L_{Q}^{0}}^{2} d s .
\end{aligned}
$$

Therefore, there are precompact sets arbitrarily close to the set $V_{\epsilon}(t)=\left\{\widehat{\Phi}_{\epsilon}(z)(t): z \in\right.$ $\left.\mathcal{B}_{q}\right\}$. Hence the set $V(t)=\left\{\widehat{\Phi}(z)(t): z \in \mathcal{B}_{q}\right\}$ is precompact in $\mathcal{H}$, Hence, the Arzelá-Ascoli shows that $\widehat{\Phi}$ is a compact multi-valued map.

Claim 4. $\widehat{\Phi}$ has a closed graph.

Let $z_{n} \longrightarrow z_{*}, \widehat{\rho}_{n} \in \widehat{\Phi} z_{n}$ and $\widehat{\rho}_{n} \longrightarrow \widehat{\rho}_{*}$ as $n \longrightarrow \infty$, we shall prove that $\widehat{\rho}_{*} \in \widehat{\Phi} z_{*} \cdot \widehat{\rho}_{n} \in \widehat{\Phi} z_{n}$ means that there exists $f_{n} \in N_{F, z_{n}+\widehat{\phi}}$ such that

$$
\widehat{\rho}_{n}(t)=\int_{0}^{t} S(t-s) f_{n}(s) d s+\int_{0}^{t} S(t-s) g(s) d B^{H}(s), t \in\left[0, t_{1}\right] .
$$

We must prove that there exists $f_{*} \in N_{F, z_{*}+\widehat{\phi}}$ such that

$$
\widehat{\rho}_{*}(t)=\int_{0}^{t} S(t-s) f_{*}(s) d s+\int_{0}^{t} S(t-s) g(s) d B^{H}(s), t \in\left[0, t_{1}\right] .
$$

Consider the linear continuous operator

$$
\Gamma: L^{2}\left(\left[0, t_{1}\right], \mathcal{H}\right) \longrightarrow \mathcal{D}_{t_{0}}^{0}, \Gamma(f)(t)=\int_{0}^{t} S(t-s) f(s) d s .
$$

From lemma (2.6), it follows that $\Gamma \circ N_{F}$ is a closed graph operator. Moreover, we have that

$$
\rho_{n}(t)-\int_{0}^{t} S(t-s) g(s) d B^{H}(s) \in \Gamma\left(N_{F, z_{n}+\widehat{\phi}}^{1}\right) .
$$

Since $z_{n} \longrightarrow z_{*}$ and $\widehat{\rho}_{n} \longrightarrow \widehat{\rho}_{*}$, there is $f_{*} \in N_{F, z_{*}+\widehat{\phi}}$ such that

$$
\widehat{\rho}_{*}(t)=\int_{0}^{t} S(t-s) f_{*}(s) d s+\int_{0}^{t} S(t-s) g(s) d B^{H}(s), \quad t \in\left[0, t_{1}\right] .
$$

Therefore $\widehat{\Phi}$ is a completely continuous

Claim 5. There exist a priori bounds on solutions. 
Let $z$ be a possible solution of the equation $z \in \lambda \widehat{\Phi}(z)$ and $z_{0}=\phi$, for some $\lambda \in(0,1)$. Then,

$$
\begin{aligned}
E|z(t)|^{2} & =E\left|\int_{0}^{t} S(t-s) f(s) d s+\int_{0}^{t} S(t-s) g(s) d B^{H}(s)\right|^{2}, t \in\left[0, t_{1}\right] . \\
& \leq 2 M t_{1} \int_{0}^{t} \eta(s) p\left(\left\|z_{s}+\widehat{\phi}_{s}\right\|_{\mathcal{D}}^{2}\right)+4 M H t_{1}^{2 H-1} \int_{0}^{t_{1}}\|g(s)\|_{L_{Q}^{0}}^{2} d s, t \in\left[0, t_{1}\right] \\
E|z(t)|^{2} & \leq 2 M t_{1} \int_{0}^{t} \eta(s) p\left(\left\|z_{s}+\widehat{\phi}_{s}\right\|_{\mathcal{D}}^{2}\right)+4 M H t_{1}^{2 H-1} \int_{0}^{t_{1}}\|g(s)\|_{L_{Q}^{0}}^{2} d s, t \in\left[0, t_{1}\right] .
\end{aligned}
$$

But

$$
\begin{aligned}
\left\|z_{t}+\widehat{\phi}_{t}\right\|_{\mathcal{D}}^{2} & \leq 2\left(\left\|z_{t}\right\|_{\mathcal{D}}^{2}+\left\|\widehat{\phi}_{t}\right\|_{\mathcal{D}}^{2}\right. \\
& \leq 4 \widetilde{K}^{2} \sup _{s \in\left[0, t_{1}\right]} E|z(s)|^{2}+4 \widetilde{K}^{2} M E|\widehat{\phi}(0)|^{2}+4 \widetilde{M}^{2}\|\phi\|_{\mathcal{D}}^{2}
\end{aligned}
$$

If we set $w_{0}(t)$ the right hand side of the above inequality we have that

and therefore (3.5) becomes

$$
\left\|z_{t}+\widehat{\phi}_{t}\right\|_{\mathcal{D}}^{2} \leq w_{0}(t)
$$

$E|z(t)|^{2} \leq 2 M t_{1} \int_{0}^{t} \eta(s) p\left(w_{0}(s)\right)+4 M H t_{1}^{2 H-1} \int_{0}^{t_{1}}\|g(s)\|_{L_{Q}^{0}}^{2} d s, t \in\left[0, t_{1}\right]$.

Using (3.6) in the definition of $w_{0}$, we have that

$$
\begin{aligned}
w_{0}(t) \leq & 4 \widetilde{K}^{2}\left[2 M t_{1} \int_{0}^{t} \eta(s) p\left(w_{0}(s)\right)+4 M H t_{1}^{2 H-1} \int_{0}^{t_{1}}\|g(s)\|_{L_{Q}^{0}}^{2} d s\right] \\
& +4 \widetilde{K}^{2} M E|\widehat{\phi}(0)|^{2}+4 \widetilde{M}^{2}\|\widehat{\phi}\|_{\mathcal{D}}^{2}, t \in\left[0, t_{1}\right] .
\end{aligned}
$$

Denoting by $v_{0}(t)$ the right-hand side of the above inequality we have

$$
\begin{gathered}
w_{0}(t) \leq v_{0}(t) \quad t \in\left[0, t_{1}\right] \\
\text { and } \left.v_{0}(0)=16 \widetilde{K}^{2} M H t_{1}^{2 H-1} \int_{0}^{t_{1}}\|g(s)\|_{L_{Q}^{0}}^{2} d s\right]+4 \widetilde{K}^{2} M E|\widehat{\phi}(0)|^{2}+4 \widetilde{M}^{2}\|\widehat{\phi}\|_{\mathcal{D}}^{2} \\
v_{0}^{\prime}(t)=8 \widetilde{K}^{2} M t_{1} \eta(t) p\left(w_{0}(t)\right) \quad t \in\left[0, t_{1}\right] .
\end{gathered}
$$

By using the nondecreasing character of $p$ we obtain

$$
v_{0}^{\prime}(t) \leq 8 \widetilde{K}^{2} M t_{1} \eta(t) p\left(v_{0}(t)\right) \quad t \in\left[0, t_{1}\right] .
$$

Then for each $t \in\left[0, t_{1}\right]$ we have

$$
\Gamma_{1}\left(v_{0}(t)\right)=\int_{v_{0}(0)}^{v_{0}(t)} \frac{d u}{p(u)} \leq 8 \widetilde{K}^{2} M t_{1} \int_{0}^{1} \eta(s) d s<\int_{v_{0}(0)}^{\infty} \frac{d u}{p(u)} .
$$

In view of $(\mathrm{H} 4)$, we deduce

$$
v_{0}(t) \leq \Gamma_{1}^{-1}\left(8 \widetilde{K}^{2} M t_{1} \int_{0}^{t_{1}} \eta(s) d s\right)=M_{0}^{2} .
$$


Since for every $t \in\left[0, t_{1}\right],\|z(t)\|_{\mathcal{D}_{t_{0}}^{o}} \leq M_{0}$

Set

$$
U_{0}=\left\{z \in \mathcal{D}_{t_{0}}^{0}: \sup _{t \in\left[0, t_{1}\right]}\left(E\|y(t)\|^{2}\right)^{\frac{1}{2}}\right\}<M_{0}+1 .
$$

From the choice of $U_{0}$, there is no $z \in \partial U_{0}$ such that $z \in \lambda \widehat{\Phi}(z)$ for some $\lambda \in(0,1)$. As a consequence of Lemma 3.1, we deduce that $\widehat{\Phi}$ has a fixed point $z_{0} \in \bar{U}_{0}$. Hence, $\Phi$ has a fixed point $y$ that is a solution to the problem (1.1). Denote this solution by $y_{0}$.

Step2. Consider now the problem,

$$
\begin{aligned}
& d y(t) \in\left[A y(t)+F\left(t, y_{t}\right)\right] d t+g(t) d B_{Q}^{H}(t), \quad \text { if } t \in\left(t_{1}, t_{2}\right], \\
& y\left(t_{1}^{+}\right)-y_{0}\left(t_{1}^{-}\right)=I_{1}\left(y_{0}\left(t_{1}^{-}\right)\right), \quad y(t)=y_{0}(t) \quad \text { if } t \in\left(-\infty, t_{1}\right] .
\end{aligned}
$$

Let

$$
\left.\mathcal{D}_{t_{1}}=\left\{y \in C\left(\left[t_{1}, t_{2}\right], \mathcal{H}\right): y\left(t_{1}^{+}\right) \text {exists } \sup _{t \in\left[t_{1}, t_{2}\right]} E\left(|y(t)|^{2}\right)<\infty\right)\right\}
$$

Set

$$
\mathcal{D}_{t_{1}}^{*}=\mathcal{D} \cap \mathcal{D}_{t_{0}} \cap \mathcal{D}_{t_{1}}
$$

Consider the operator $\Phi_{1}: \mathcal{D}_{t_{1}} \longrightarrow \mathcal{P}\left(\mathcal{D}_{t_{1}}\right)$ defined by,

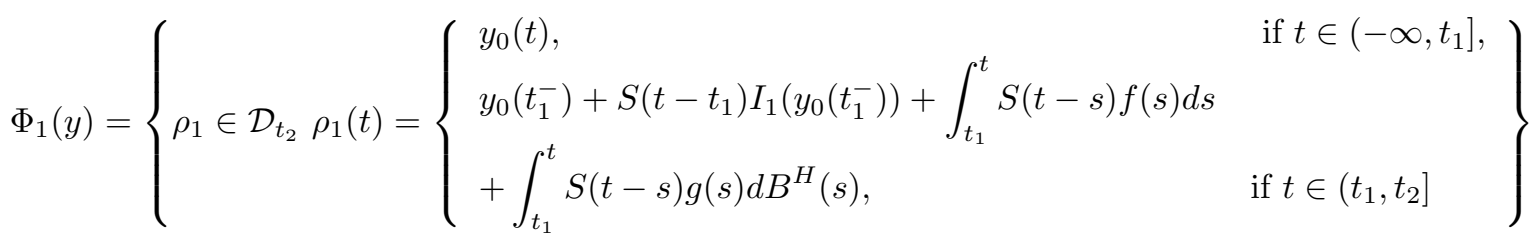

where $f \in N_{F, y}=\left\{f \in L^{2}\left(\left[t_{1}, t_{2}\right], \mathcal{H}\right): f(t) \in F\left(t, y_{t}\right)\right.$ for a.e. $\left.t \in\left[t_{1}, t_{2}\right]\right\}$.

Let $\widehat{\phi}(\cdot):\left(-\infty, t_{2}\right] \longrightarrow \mathcal{H}$ be the function defined by

$$
\widehat{\phi}(t)= \begin{cases}y_{0}(t), & \text { if; } t \in\left(-\infty, t_{1}\right], \\ y_{0}\left(t_{1}^{-}\right)+S\left(t-t_{1}\right) I_{1}\left(y_{0}\left(t_{1}^{-}\right)\right), & \text {if } t \in\left(t_{1}, t_{2}\right]\end{cases}
$$

Then $\widehat{\phi}_{t_{1}}$ is an element of $\mathcal{D}_{t_{1}}^{*}$ and $\widehat{\phi}_{t_{1}}=y_{0}$.

Let $y(t)=z(t)+\widehat{\phi}(t), t_{1}<t \leq t_{2}$. Obviously, if $y$ satisfies the integral equation $y(t)=y_{0}\left(t_{1}^{-}\right)+S\left(t-t_{1}\right) I_{1}\left(y_{0}\left(t_{1}^{-}\right)\right)+\int_{t_{1}}^{t} S(t-s) f(s) d s+\int_{t_{1}}^{t} S(t-s) g(s) d B_{Q}^{H}(s), t \in\left[t_{1}, t_{2}\right]$,

that $z$ satisfies $z\left(t_{1}\right)=0, t \in\left(-\infty, t_{1}\right]$ and

$$
z(t)=\int_{t_{1}}^{t} S(t-s) f(s) d s+\int_{t_{1}}^{t} S(t-s) g(s) d B_{Q}^{H}(s)+S\left(t-t_{1}\right) I_{1}\left(z_{0}\left(t_{1}^{-}\right)+\widehat{\phi}\left(t_{1}^{-}\right)\right), \quad t \in\left[t_{1}, t_{2}\right] .
$$

Set $\mathcal{D}_{t_{1}}^{1}=\left\{z \in \mathcal{D}_{t_{1}}^{*}, \quad\right.$ such that $\left.z_{t_{1}}=0\right\}$

Let the operator $\widehat{\Phi}: \mathcal{D}_{t_{1}}^{1} \rightarrow \mathcal{P}\left(\mathcal{D}_{t_{1}}^{1}\right)$ be defined by

$$
\widehat{\Phi}(z)=\left\{\widehat{\rho} \in \mathcal{D}_{t_{1}}^{1} \widehat{\rho}(t)=\left\{\begin{array}{ll}
0, & \text { if } ; t \in\left(-\infty, t_{1}\right], \\
\int_{t_{1}}^{t} S(t-s) f(s) d s+\int_{t_{1}}^{t} S(t-s) g(s) d B^{H}(s) & \\
+S\left(t-t_{1}\right)\left(I_{1}\left(z\left(t_{1}^{-}\right)+\phi\left(t_{1}^{-}\right)\right)\right. & \text {if } t \in\left[t_{1}, t_{2}\right] .
\end{array}\right\}\right.
$$


where

$$
f \in N_{F, z+\widehat{\phi}}=\left\{f \in L^{2}\left(\left[t_{1}, t_{2}\right], \mathcal{H}\right): f(t) \in F\left(t, z_{t_{1}}+\widehat{\phi}_{t_{1}}\right) \text { a.e. } t \in\left[t_{1}, t_{2}\right]\right\} .
$$

From (H4), for each $t \in\left[t_{1}, t_{2}\right]$, we have

$$
\begin{aligned}
E|z(t)|^{2}= & E\left|\int_{t_{1}}^{t} S(t-s) f(s) d s+\int_{t_{1}}^{t} S(t-s) g(s) d B^{H}(s)+S\left(t-t_{1}\right) I_{1}\left(z_{0}\left(t_{1}^{-}\right)+\widehat{\phi}\left(t_{1}^{-}\right)\right)\right|^{2}, \\
\leq & 2 M\left(t_{2}-t_{1}\right) \int_{t_{1}}^{t} \eta(s) p\left(\left\|z_{s}+\widehat{\phi}_{s}\right\|_{\mathcal{D}}^{2}\right) \\
& +8 M H\left(t_{2}-t_{1}\right)^{2 H-1} \int_{t_{1}}^{t_{2}}\|g(s)\|_{L_{Q}^{0}}^{2} d s \\
& +\left.4 M \sup _{t \in\left(-\infty, t_{1}\right]} E\left(I_{1}\left(z_{0}\left(t_{1}\right)\right)+\widehat{\phi}\left(t_{1}\right)\right)\right|^{2}, t \in\left[t_{1}, t_{2}\right] .
\end{aligned}
$$

If we set $w_{1}(t)$ the right hand side of the above inequality we have that

and therefore (3.11) becomes

$$
\left\|z_{t}+\widehat{\phi}_{t}\right\|_{\mathcal{D}}^{2} \leq w_{1}(t)
$$

$$
\begin{aligned}
E|z(t)|^{2} \leq & 2 M\left(t_{2}-t_{1}\right) \int_{t_{1}}^{t} \eta(s) p\left(w_{1}(s)\right)+8 M H\left(t_{2}-t_{1}\right)^{2 H-1} \int_{t_{1}}^{t_{2}}\|g(s)\|_{L_{Q}^{0}}^{2} d s \\
& +\left.4 M \sup _{t \in\left(-\infty, t_{1}\right]} E\left(I_{1}\left(z_{0}\left(t_{1}\right)\right)+\widehat{\phi}\left(t_{1}\right)\right)\right|^{2}, t \in\left[t_{1}, t_{2}\right] .
\end{aligned}
$$

Using (3.12) in the definition of $w_{1}$, we have that

$$
\begin{aligned}
w_{1}(t) \leq & 4 \widetilde{K}^{2}\left[2 M\left(t_{2}-t_{1}\right) \int_{t_{1}}^{t} \eta(s) p\left(w_{1}(s)\right)+8 M H\left(t_{2}-t_{1}\right)^{2 H-1} \int_{t_{1}}^{t_{2}}\|g(s)\|_{L_{Q}^{0}}^{2} d s,\right. \\
& +4 M \sup _{t \in\left(-\infty, t_{1}\right]} E\left(I_{1}\left(z_{0}\left(t_{1}\right)\right)\right]+4 M \widetilde{K}^{2} E|\widehat{\phi}(0)|^{2}+4 \widetilde{M} \widetilde{M}^{2}\|\widehat{\phi}\|_{\mathcal{D}}^{2}, t \in\left[t_{1}, t_{2}\right], \\
= & N_{1}+8 \widetilde{K}^{2} M\left(t_{2}-t_{1}\right) \int_{t_{1}}^{t} \eta(s) p\left(w_{1}(s)\right) .
\end{aligned}
$$

Denoting by $v_{1}(t)$ the right-hand side of the above inequality we have

$$
\begin{gathered}
w_{1}(t) \leq v_{1}(t) \quad t \in\left[t_{1}, t_{2}\right], \\
v_{1}\left(t_{1}\right)=N_{1}
\end{gathered}
$$

and

$$
v_{1}^{\prime}(t)=8 \widetilde{K}^{2} M\left(t_{2}-t_{1}\right) \eta(t) p\left(w_{1}(t)\right) \quad t \in\left[t_{1}, t_{2}\right] .
$$

By using the nondecreasing character of $p$ get

$$
v_{1}^{\prime}(t) \leq 8 \widetilde{K}^{2} M\left(t_{2}-t_{1}\right) \eta(t) p\left(v_{1}(t)\right) \quad t \in\left[t_{1}, t_{2}\right] .
$$

Then for each $t \in\left[0, t_{1}\right]$ we have

$$
\int_{N_{1}}^{v_{1}(t)} \frac{d u}{p(u)} \leq 8 \widetilde{K}^{2} M\left(t_{2}-t_{1}\right) \int_{t_{1}}^{t_{2}} \eta(s) d s
$$


In view of $(\mathrm{H} 4)$, we obtain

$$
v_{1}(t) \leq \Gamma_{2}^{-1}\left(8 \widetilde{K}^{2} M\left(t_{2}-t_{1}\right) \int_{t_{1}}^{t_{2}} \eta(s) d s\right)=M_{1}^{2} .
$$

Since for every $t \in\left[t_{1}, t_{2}\right],\|z(t)\|_{\mathcal{D}_{t_{1}}^{2}} \leq M_{1}$

Set

$$
U_{1}=\left\{z \in \mathcal{D}_{t_{1}}^{1}: \sup _{t \in\left[t_{1}, t_{2}\right]}\left(E\|z(t)\|^{2}\right)^{\frac{1}{2}}\right\}<M_{1}+1 .
$$

As in Step 1 we can show that $\widehat{\Phi}: \bar{U}_{1} \longrightarrow \mathcal{P}_{c v}\left(\mathcal{D}_{t_{1}}^{1}\right)$ is a compact multivalued map and u.s.c.

From the choice of $U_{1}$, there is no $z \in \partial U_{1}$ such that $z \in \lambda \widehat{\Phi}(z)$ for some $\lambda \in(0,1)$. As a consequence of Lemma 3.1, we deduce that $\widehat{\Phi}$ has a fixed point $z_{1} \in \bar{U}_{1}$. Hence, $\Phi$ has a fixed point $y$ that is a solution to the problem (1.1). Denote this solution by $y_{1}$.

Step3. We continue this process and take into account that $z_{m}:=\left.z\right|_{\in\left[t_{m}, T\right]}$ is a fixed point of the operator $\widehat{\Phi}_{m}$ defined by

Let the operator $\widehat{\Phi}: \mathcal{D}_{t_{m}}^{m} \rightarrow \mathcal{P}\left(\mathcal{D}_{t_{m}}^{m}\right)$ defined by

$$
\widehat{\Phi}(z)=\left\{\widehat{\rho} \in \mathcal{D}_{t_{m}}^{m} \widehat{\rho}(t)=\left\{\begin{array}{ll}
0, & \text { if } t \in\left(-\infty, t_{m}\right], \\
\int_{t_{m}}^{t} S(t-s) f(s) d s+\int_{t_{m}}^{t} S(t-s) g(s) d B^{H}(s) & \\
+S\left(t-t_{m}\right)\left(I_{m}\left(z_{m-1}\left(t_{m}^{-}\right)+\phi\left(t_{m}^{-}\right)\right)\right. & \text {if } t \in\left[t_{m}, T\right] .
\end{array}\right\}\right.
$$

where

$$
f \in N_{z+\widehat{\phi}}=\left\{f \in L^{2}\left(\left[t_{m}, T\right], \mathcal{H}\right): f(t) \in F\left(t, z_{t_{t}}+\widehat{\phi}_{t}\right) \text { a.e. } t \in\left[t_{m}, T\right]\right\} .
$$

Let

$$
\left.\mathcal{D}_{t_{m}}=\left\{z \in\left(\left[t_{m}, T\right], \mathcal{H}\right), z\left(t_{m}^{+}\right) \text {existe } \sup _{t \in\left[t_{m}, T\right]} E\left(|y(t)|^{2}\right)<\infty\right)\right\}
$$

Set

$$
\mathcal{D}_{t_{m}}^{*}=\mathcal{D} \cap \mathcal{D}_{m-1} \cap \mathcal{D}_{m}
$$

and $\mathcal{D}_{t_{m}}^{m}=\left\{z \in \mathcal{D}_{t_{m}}^{*}, \quad\right.$ such that $\left.z_{t_{m}}=0\right\}$

Then, there exists a fixed point $z_{m}$ of $\widehat{\Phi}_{m}$, The fixed point $z$ of the operator $\widehat{\Phi}$ is then defined by

$$
z(t)= \begin{cases}z_{0}(t), & \text { if } t \in\left(-\infty, t_{1}\right], \\ z_{1}(t), & \text { if } t \in\left(t_{1}, t_{2}\right], \\ \cdot & \\ \cdot & \\ z_{m}(t), & \text { if } t \in\left(t_{m}, t_{m+1}\right],\end{cases}
$$

Hence, $\Phi$ has a fixed point $y$ that is a solution to the problem (1.1). This completes the proof. 
3.2. The nonconvex case. In this section we present a result for the problem (1.1) in the spirit of the nonlinear alternative of Leray-Schauder type (Lemma 2.8), for single-valued maps, combined with a selection theorem due to Bressan and Colombo [10] for lower semicontinuous multivalued maps with decomposable values.

Let $\mathcal{A}$ be a subset of $J \times \mathcal{D}$. $\mathcal{A}$ is $\mathcal{L} \otimes \mathcal{B}$ measurable if $\mathcal{A}$ belongs to the $\sigma$-algebra generated by all sets of the form $\mathcal{J} \times \mathcal{D}$ where $\mathcal{J}$ is Lebesgue measurable in $J$ and $\mathcal{D}$ is Borel measurable in $\mathcal{D}$. A subset $\mathcal{A}$ of $L^{2}(J, \mathcal{H})$ is decomposable if for all $w, v \in \mathcal{A}$ and $\mathcal{J} \in J$ measurable, $w \chi_{\mathcal{J}}+v \chi_{J-\mathcal{J}} \in A$, where $\chi$ stands for the characteristic function.

Let $G: \mathcal{H} \longrightarrow \mathcal{P}(\mathcal{H})$ be a multivalued operator with nonempty closed values. $G$ is lower semi-continuous (l.s.c.) if the set $\{y \in \mathcal{H}: G(y) \cap B \neq \emptyset\}$ is open for any open set $B$ in $\mathcal{H}$.

Definition 3.4. Let $Y$ be a separable metric space and let $N: Y \longrightarrow \mathcal{P}\left(L^{2}(J, \mathcal{H})\right.$ be a multivalued operator. We say that $N$ has property $(B C)$ if

1): $N$ is lower semi-continuous (l.s.c.);

2): $N$ has nonempty closed and decomposable values.

Let $F: J \times \mathcal{D} \longrightarrow \mathcal{P}(\mathcal{H})$ ) be a multivalued map with nonempty compact values. Assign to $\mathrm{F}$ the multivalued operator

$$
\Psi: D_{T}^{*} \longrightarrow \mathcal{P}\left(L^{2}(J, \mathcal{H})\right)
$$

by letting

$$
\Psi(y)=\left\{f \in L^{2}(J, \mathcal{H}): f(t) \in F\left(t, y_{t}\right) \text { for a.e. } t \in J\right\} .
$$

The operator $\Psi$ is called the Niemytzki operator associated to $F$.

Definition 3.5. Let $F: J \times \mathcal{H} \longrightarrow \mathcal{P}(\mathcal{H})$ be a multivalued function with nonempty compact values. We say $F$ is of lower semi-continuous type (l.s.c. type) if its associated Niemytzki operator $\Psi$ is lower semi-continuous and has nonempty closed and decomposable values.

Next we state a selection theorem due to Bressan and Colombo [10].

Theorem 3.6. Let $Y$ be a separable metric space and let $N: Y \longrightarrow \mathcal{P}\left(L^{2}(J, \mathcal{H})\right)$ be a multivalued operator which has property $(B C)$. Then $N$ has a continuous selection, i.e. there exists a continuous function (single-valued) $\widetilde{g}: Y \longrightarrow L^{2}(J, \mathcal{H})$ such that $\widetilde{g}(y) \in N(y)$ for every $y \in Y$.

Let us introduce the following hypotheses which are assumed hereafter:

(H6): $F: J \times \mathcal{D} \longrightarrow \mathcal{P}(\mathcal{H})$ is a nonempty compact valued multivalued map such that:

a): $(t, y) \longmapsto F(t, y)$ is $\mathcal{L} \otimes \mathcal{B}$ measurable and for every $t \in J$, the multifunction $t \rightarrow F\left(t, y_{t}\right)$ is measurable.

b): $(t, y) \longmapsto F(t, y)$ is lower semi-continuous for a.e. $t \in J$.

(H7): $\left.E|F(t, \Theta)|^{2}=\sup \left\{E(|f|)^{2}: f \in F(t, \Theta)\right\}\right\} \leq \eta(t) p\left(\|\Theta\|_{\mathcal{D}}^{2}\right), \quad t \in$ $J, \quad \Theta \in \mathcal{D}$,

where $\eta \in L^{2}\left(J, \mathbb{R}^{+}\right)$and $p: \mathbb{R}^{+} \longrightarrow(0, \infty)$ is continuous and increasing with

$$
8 \widetilde{K}^{2} M T \int_{t_{0}}^{T} \eta(s) d s \leq \int_{N}^{\infty} \frac{d u}{p(u)},
$$




$$
N=4 \widetilde{K}^{2}\left[4 M m \sum_{k=1}^{m} c_{k}+8 \widetilde{K}^{2} M T^{2 H-1} \int_{0}^{T}\|g(s)\|_{L_{Q}^{0}}^{2} d s\right]+C .
$$

The following lemma is crucial in the proof of our main theorem.

Lemma 3.7. [17] Let $F: J \times \mathcal{D} \longrightarrow \mathcal{P}_{c p}(\mathcal{H})$ be a multivalued map. Assume that (H6) and $(H 7)$ hold. Then $F$ is of l.s.c. type.

Theorem 3.8. Assume that hypotheses $(H 1)-(H 3)$ and $(H 6)-(H 7)$ hold. Then the impulsive initial value problem (1.1) has at least one solution.

Proof. Consider the operator $G: \mathcal{D}_{T}^{*} \rightarrow \mathcal{P}\left(\mathcal{D}_{T}^{*}\right)$ defined by

$$
G(y)= \begin{cases}\phi(t), & \text { if } t \in(-\infty, 0], \\ S(t) \phi(0)+\int_{0}^{t} S(t-s) f(s) d s & \\ +\int_{0}^{t} S(t-s) g(s) d B^{H}(s)+\sum_{0<t_{k}<t} S\left(t-t_{k}\right) I_{k}\left(y\left(t_{k}^{-}\right)\right), & \text {if } t \in[0, T]\end{cases}
$$

Let $\widehat{\phi}:(-\infty, T] \longrightarrow \mathcal{H}$ be the function defined by

$$
\widehat{\phi}(t)= \begin{cases}\phi(t), & t \in(-\infty, 0], \\ S(t) \phi(0), & t \in[0, T] ;\end{cases}
$$

Then $\widehat{\phi}$ is an element of $\mathcal{D}_{T}^{*}$ and $\widehat{\phi}_{0}=\phi$. It is evident that $z$ satisfies $z_{0}=$ $0, t \in(-\infty, 0]$ and

$$
\begin{aligned}
z(t)= & \int_{0}^{t} S(t-s) f(s) d s+\int_{0}^{t} S(t-s) g(s) d B_{Q}^{H}(s) \\
& +\sum_{0<t_{k}<t} S\left(t-t_{k}\right) I_{k}\left(z\left(t_{k}^{-}\right)+\widehat{\phi}\left(t_{k}^{-}\right)\right), t \in J
\end{aligned}
$$

where $f(t) \in F\left(t, z_{t}+\widehat{\phi}_{t}\right)$ for a.e. $t \in[0, T]$.

Set $\mathcal{D}_{T}^{* *}=\left\{z \in \mathcal{D}_{T}^{*}, \quad\right.$ such that $\left.z_{0}=0 \in \mathcal{D}\right\}$ and for any $z \in \mathcal{D}_{T}^{* *}$ we have

$$
\|z\|_{\mathcal{D}_{T}^{* *}}=\left\|z_{0}\right\|_{\mathcal{D}}+\sup _{t \in[0, T]}\left(E\|z(t)\|^{2}\right)^{\frac{1}{2}}=\sup _{t \in[0, T]}\left(E\|z(t)\|^{2}\right)^{\frac{1}{2}}
$$

thus $\left(\mathcal{D}_{T}^{* *},\|\cdot\|_{\mathcal{D}_{T}^{* *}}\right)$ is a Banach space.

We define the Niemytzki operator associated to $F$,

$$
\Psi: \mathcal{D}_{T}^{* *} \longrightarrow \mathcal{P}\left(L^{2}(J, \mathcal{H})\right)
$$

by letting

$$
\Psi(z)=\left\{f \in L^{2}(J, \mathcal{H}): f(t) \in F\left(t, z_{t}+\widehat{\phi}_{t}\right) \text { for a.e. } t \in J\right\}
$$

be a selection set of $\Psi$. From $(H 6)$ and $(H 7)$ imply by Lemma 3.7 that $F$ is of lower semi-continuous type. Then from Theorem 3.6 there exists a continuous function $f: \mathcal{D}_{T}^{* *} \longrightarrow L^{2}(J, \mathcal{H})$ such that $f(z) \in \Psi(z+\widehat{\phi})$ for all $z \in \mathcal{D}_{T}^{* *}$.

Consider the problem,

$$
\begin{cases}d z(t) \in\left[A z(t)+f\left(z_{t}+\widehat{\phi}_{t}\right)\right] d t+g(t) d B_{Q}^{H}(t), & t \in J=[0, T], t \neq t_{k}, \\ z\left(t_{k}^{+}\right)-z\left(t_{k}^{-}\right)=I_{k}\left(z\left(t_{k}^{-}\right)+\widehat{\phi}\left(t_{k}^{-}\right)\right), k=1, \ldots, m, & \\ z(t)=0, & J_{0}=(-\infty, 0],\end{cases}
$$


and consider the operator $\widehat{G}_{1}: \mathcal{D}_{T}^{* *} \rightarrow \mathcal{D}_{T}^{* *}$ defined by

$$
\widehat{G}_{1}(z)=\left\{\begin{array}{ll}
0, & \text { if } ; t \in(-\infty, 0], \\
\int_{0}^{t} S(t-s) f\left(z_{s}+\widehat{\phi}_{s}\right) d s+\int_{0}^{t} S(t-s) g(s) d B^{H}(s) & \\
+\sum_{0<t_{k}<t} S\left(t-t_{k}\right) I_{k}\left(z\left(t_{k}^{-}\right)+\widehat{\phi}\left(t_{k}^{-}\right)\right), & \text {if } t \in[0, T],
\end{array}\right\} .
$$

where $f(z) \in \Psi(z+\widehat{\phi})$.

Clearly, if $z \in \mathcal{D}_{T}^{* *}$ is fixed point of $\widehat{G}_{1}$, then $z$ is fixed fixed point of $\widehat{G}$. Thus, there exists $y \in \mathcal{D}_{T}^{*}$ such that $y$ is a fixed point of the operator $G$,

Clearly, $\widehat{G}_{1}$ is completely continuous and there exists $M_{T}>0$ such that for every solution of $z=\lambda \widehat{G}_{1}(z)$, for some $\lambda \in(0,1)$, we have $\|z\|_{\mathcal{D}_{T}^{* *}} \leq M_{T}$. Set

$$
U=\left\{z \in \mathcal{D}_{T}^{* *}:\|z\|_{\mathcal{D}_{T}^{* *}}<M_{T}+1\right\} .
$$

We see that $U$ is an open set in $\mathcal{D}_{T}^{* *}$. From the choice of $U$ there is no $z \in \partial U$ such that $z=\lambda \widehat{G}_{1}(z)$ for some $\lambda \in(0,1)$. As a consequence of the nonlinear alternative of Leray Schauder type lemma 2.8, we deduce that $\widehat{G}_{1}(z)$ has a fixed point $z$ in $\bar{U}$. Hence, $G$ has a fixed point $y$ that is a solution to the problem $(1.1)$

Now we present a second result for the problem (1.1) with a nonconvex valued right-hand side. Our considerations are based on a fixed point theorem for contraction multivalued operators given by Covitz and Nadler in 1970 (see also Deimling [16], Theorem 11.1).

Lemma 3.9. [16] Let $(X, d)$ be a complete metric space. If the multivalued operator $G: X \longrightarrow \mathcal{P}_{c l}(X)$ is a contraction, then $G$ has at least one fixed point.

Let us introduce the following hypotheses:

(H8): $F: J \times \mathcal{D} \longrightarrow \mathcal{P}_{c p}(\mathcal{H}) ;(t, y) \longrightarrow F(t, y)$ is measurable for each $y \in \mathcal{D}$.

(H9): There exists a function $l_{f} \in L^{2}\left(J, \mathbb{R}^{+}\right)$(denote $\left.l^{*}=\int_{0}^{T} l^{2}(t) d t\right)$, such that

$$
E H_{d}^{2}(F(t, x), F(t, y)) \leq l_{f}(t)\|x-y\|_{\mathcal{D}}^{2} \text { for each } x, y \in \mathcal{D}, t \in J .
$$

Theorem 3.10. Assume that hypotheses (H1), (H2) and (H8) hold. Then the problem (1.1) has at least one mild solution.

Although the proof follows the same steps than the one of theorem 3.8, the technical details necessary for the proof are different.

Proof. The proof will be given in several steps.

Consider the problem $(1.1)$ on $\left(-\infty, t_{1}\right]$

$$
\begin{array}{ll}
d y(t) \in\left[A y(t)+F\left(t, y_{t}\right)\right] d t+g(t) d B_{Q}^{H}(t), & \text { if } t \in\left[0, t_{1}\right], \\
y(t)=\phi(t), & \text { if } t \in(-\infty, 0] .
\end{array}
$$

Let

$$
\mathcal{D}_{t_{0}}=\left\{y \in C\left(\left[0, t_{1}\right], \mathcal{H}\right): \sup _{t \in\left[0, t_{1}\right]} E\left(|y(t)|^{2}\right)<\infty\right\}
$$

Set

$$
\mathcal{D}_{t_{0}}^{*}=\mathcal{D} \cap \mathcal{D}_{t_{0}}
$$


We transform the problem (3.2) into a fixed point problem. Consider the multivalued operator $\Phi: \mathcal{D}_{t_{0}}^{*} \rightarrow \mathcal{P}\left(\mathcal{D}_{t_{0}}^{*}\right)$ defined by

$$
\Phi(y)=\left\{\rho \in \mathcal{D}_{t_{0}}^{*}: \rho(t)=\left\{\begin{array}{ll}
\phi(t), & \text { if } t \in(-\infty, 0], \\
S(t) \phi(0)+\int_{0}^{t} S(t-s) f(s) d s & \\
+\int_{0}^{t} S(t-s) g(s) d B^{H}(s), & \text { if } t \in\left[0, t_{1}\right]
\end{array}\right\}\right.
$$

where $f \in N_{F, y}=\left\{f \in L^{2}\left(\left[0, t_{1}\right], \mathcal{H}\right): f(t) \in F\left(t, y_{t}\right)\right.$ for a.e. $\left.t \in\left[0, t_{1}\right]\right\}$.

We will prove that $\Phi$ has a fixed point.

Let $\widehat{\phi}:\left(-\infty, t_{1}\right] \longrightarrow \mathcal{H}$ be the function defined by

$$
\widehat{\phi}(t)= \begin{cases}\phi(t), & t \in(-\infty, 0], \\ S(t) \phi(0), & t \in\left[0, t_{1}\right]\end{cases}
$$

Then $\widehat{\phi}$ is an element of $\mathcal{D}_{t_{0}}^{*}$ and $\widehat{\phi}_{0}=\phi$. Let $y(t)=z(t)+\widehat{\phi}(t),-\infty<t \leq t_{1}$.

Obviously, if $y$ satisfies the integral equation

$$
y(t)=S(t) \phi(0)+\int_{0}^{t} S(t-s) f(s) d s+\int_{0}^{t} S(t-s) g(s) d B_{Q}^{H}(s), t \in\left[0, t_{1}\right],
$$

then $z$ satisfies $z_{0}=0, t \in(-\infty, 0]$ and

$$
z(t)=\int_{0}^{t} S(t-s) f(s) d s+\int_{0}^{t} S(t-s) g(s) d B_{Q}^{H}(s), t \in\left[0, t_{1}\right],
$$

where $f(t) \in F\left(t, z_{t}+\widehat{\phi}_{t}\right)$ for a.e. $t \in\left[0, t_{1}\right]$.- $\operatorname{Set} \mathcal{D}_{t_{0}}^{0}=\left\{z \in \mathcal{D}_{t_{0}}^{*}, \quad\right.$ such that $\left.\quad z_{0}=0 \in \mathcal{D}\right\}$ and for any $z \in \mathcal{D}_{t_{0}}^{0}$ we have

$$
\|y\|_{\mathcal{D}_{t_{0}}^{o}}=\left\|z_{0}\right\|_{\mathcal{D}}+\sup _{t \in\left[0, t_{1}\right]}\left(E\|z(t)\|^{2}\right)^{\frac{1}{2}}=\sup _{t \in\left[0, t_{1}\right]}\left(E\|y(t)\|^{2}\right)^{\frac{1}{2}}
$$

thus $\left(\mathcal{D}_{t_{0}}^{0},\|\cdot\|_{\mathcal{D}_{t_{0}}^{0}}\right)$ is a Banach space. Let the operator $\widehat{\Phi}: \mathcal{D}_{t_{0}}^{0} \rightarrow \mathcal{P}\left(\mathcal{D}_{t_{0}}^{0}\right)$ defined by

$$
\widehat{\Phi}(z)=\left\{\widehat{\rho} \in \mathcal{D}_{t_{0}}^{0}: \widehat{\rho}(t)=\left\{\begin{array}{ll}
0, & \text { if } ; \in(-\infty, 0], \\
\int_{0}^{t} S(t-s) f(s) d s+\int_{0}^{t} S(t-s) g(s) d B^{H}(s) & \text { if } t \in\left[0, t_{1}\right] .
\end{array}\right\}\right.
$$

We shall show that $\widehat{\Phi}$ satisfies the assumptions of Lemma 3.9. The proof will be given in two claims.

Claim 1. $\widehat{\Phi}(z) \in \mathcal{P}_{c l}\left(\mathcal{D}_{t_{0}}^{0}\right)$ for each $z \in \mathcal{D}_{t_{0}}^{0}$.

Let $z_{n} \in \widehat{\Phi}(z)$ and $\left\|z_{n}-z\right\|_{\mathcal{D}_{t_{0}}^{o}}^{2} \longrightarrow 0 . \quad z \in \mathcal{D}_{t_{0}}^{0}$ and there exist $f \in N_{F, z+\widehat{\phi}}$, such that

$$
z_{n}(t)=\int_{0}^{t} S(t-s) f_{n}(s) d s+\int_{0}^{t} S(t-s) g(s) d B_{Q}^{H}(s) .
$$

Since $F(t, z(t)+\widehat{\phi}(t))$ is compact values and from (H8), we may pass to a subsequence if necessary to get that $f_{n}$ converges to $f$ in $L^{2}(J, \mathcal{H})$. Then, for each $t \in\left[0, t_{1}\right]$ 


$$
\begin{aligned}
E\left|z_{n}(t) \quad-\int_{0}^{t} S(t-s) f(s) d s-\int_{0}^{t} S(t-s) g(s) d B_{Q}^{H}(s)\right|^{2} \\
\longrightarrow 0, \text { as } n \longrightarrow 0 .
\end{aligned}
$$

So we have that there exists a $f(\cdot) \in N_{F, z+\widehat{\phi}}$ such that

$$
z(t)=\int_{0}^{t} S(t-s) f(s) d s+S(t-s) g(s) d B_{Q}^{H}(s)
$$

which implies $z \in \widehat{G}(z)$.

Cliam 2. There exists $\gamma<1$, such that

$$
E H_{d}^{2}\left(\widehat{\Phi}\left(z_{1}\right), \widehat{\Phi}\left(z_{2}\right)\right) \leq \gamma\left\|z_{1}-z_{2}\right\|_{\mathcal{D}_{t_{0}}^{0}}
$$

for any $z_{1}, z_{2} \in \mathcal{D}_{t_{0}}^{0}$. For all $h_{1} \in \widehat{\Phi}\left(z_{1}\right)$, there exists $f_{1}(\cdot) \in N_{F, z_{1}+\widehat{\phi}}$, such that

$$
h_{1}(t)=\int_{0}^{t} S(t-s) f_{1}(s) d s+\int_{0}^{t} S(t-s) g(s) d B_{Q}^{H}(s)
$$

For $H_{d}\left(F\left(t, z_{1}(t)+\widehat{\phi}(t)\right), F\left(t, z_{2}(t)+\widehat{\phi}\right)\right) \leq l(t)\left|z_{1}(t)-z_{2}(t)\right|$, there exists $f_{2}(t) \in N_{F, z_{2}}$, such that

$$
E\left|f_{1}(t)-f_{2}(t)\right|^{2} \leq l_{f}(t)\left\|z_{1}(t)-z_{2}(t)\right\| \text {, a.e. } t \in\left[0, t_{1}\right] .
$$

Define

$$
h_{2}(t)=\int_{0}^{t} S(t-s) f_{2}(s) d s+\int_{0}^{t} S(t-s) g(s) d B_{Q}^{H}(s)
$$

and we have

$$
\begin{aligned}
E\left|h_{1}(t)-h_{2}(t)\right|^{2} & \leq E\left|\int_{0}^{t} S(t-s)\left(f_{1}(s)-f_{2}(s)\right) d s\right|^{2} \\
& \leq E\left|\int_{0}^{t} S(t-s)\left(f_{1}(s)-f_{2}(s)\right) d s\right|^{2} \\
& \leq M T \int_{0}^{t} l^{2}(s) E\left|z_{1}-z_{2}\right|^{2} d s \\
& \leq \int_{0}^{t} \bar{l}(s) E\left|z_{1}-z_{2}\right|^{2} d s \\
& \leq \frac{1}{\tau} e^{\tau L(t)}\left\|z_{1}-z_{2}\right\|_{*}^{2} .
\end{aligned}
$$

Thus,

Therefore,

$$
e^{-\tau L(t)} E\left|h_{1}(t)-h_{2}(t)\right|^{2} \leq \frac{1}{\tau}\left\|z_{1}-z_{2}\right\|_{*}^{2}
$$

$$
\left\|h_{1}(t)-h_{2}(t)\right\|_{*}^{2} \leq \frac{1}{\tau}\left\|z_{1}-z_{2}\right\|_{*}^{2},
$$

where $L(t)=\int_{0}^{t} L(s) d s, \bar{l}(t)=M T l^{2}(t)$ and $\|\cdot\|_{*}$ denote the Bielecki-type [9] norm on $C\left(\left[0, t_{1}\right], X\right)$ defined by

$$
\|y\|_{*}^{2}=\sup _{t \in\left[0, t_{1}\right]} E|y(t)|^{2} e^{-\tau L(t)}, \tau>1
$$

By an analogous relation, obtained by interchanging the roles of $z_{1}$ and $z_{2}$, it follows that 


$$
E H_{d}^{2}\left(\widehat{\Phi}\left(z_{1}\right)-\widehat{\Phi}\left(z_{2}\right)\right) \leq \frac{1}{\tau}\left\|z_{1}-z_{2}\right\|_{*}^{2}
$$

So, $\widehat{\Phi}$ is a contraction, and thus, by Lemma $3.9, \widehat{\Phi}$ has a fixed point $z$, so the problem (3.15) has at least one solution. Denote this solution by $y_{0}$. Step2. Consider now the problem,

$$
\begin{array}{ll}
d y(t) \in\left[A y(t)+F\left(t, y_{t}\right)\right] d t+g(t) d B_{Q}^{H}(t), & \text { if } t \in\left(t_{1}, t_{2}\right], \\
y\left(t_{1}^{+}\right)-y_{0}\left(t_{1}^{-}\right)=I_{1}\left(y_{0}\left(t_{1}^{-}\right)\right), \quad y(t)=y_{0}(t) & \text { if; } t \in\left(-\infty, t_{1}\right] .
\end{array}
$$

Let

$$
\left.\mathcal{D}_{t_{1}}=\left\{y \in C\left(\left[t_{1}, t_{2}\right], \mathcal{H}\right): y\left(t_{1}^{+}\right) \text {exists } \sup _{t \in\left[t_{1}, t_{2}\right]} E\left(|y(t)|^{2}\right)<\infty\right)\right\}
$$

Set

$$
\mathcal{D}_{t_{1}}^{*}=\mathcal{D} \cap \mathcal{D}_{t_{0}} \cap \mathcal{D}_{t_{1}}
$$

Consider the operator $\Phi_{1}: \mathcal{D}_{t_{1}}^{*} \longrightarrow \mathcal{P}\left(\mathcal{D}_{t_{1}}^{*}\right)$ defined by,

$$
\Phi_{1}(y)=\left\{\rho_{1} \in \mathcal{D}_{t_{1}}^{*}: \rho_{1}(t)=\left\{\begin{array}{ll}
y_{0}(t), & \text { if } t \in\left(-\infty, t_{1}\right], \\
y_{0}\left(t_{1}^{-}\right)+S\left(t-t_{1}\right) I_{1}\left(y_{0}\left(t_{1}^{-}\right)\right)+\int_{t_{1}}^{t} S(t-s) f(s) d s & \\
+\int_{t_{1}}^{t} S(t-s) g(s) d B^{H}(s), & \text { if } t \in\left(t_{1}, t_{2}\right]
\end{array}\right\}\right.
$$

where $f \in N_{F, y}=\left\{f \in L^{2}\left(\left[t_{1}, t_{2}\right], \mathcal{H}\right): f(t) \in F\left(t, y_{t}\right)\right.$ for a.e.t $\left.\in\left[t_{1}, t_{2}\right]\right\}$.

Let $\widehat{\phi}(\cdot):\left(-\infty, t_{2}\right] \longrightarrow \mathcal{H}$ be the function defined by

$$
\widehat{\phi}(t)= \begin{cases}y_{0}(t), & \text { if; } t \in\left(-\infty, t_{1}\right], \\ y_{0}\left(t_{1}^{-}\right)+S\left(t-t_{1}\right) I_{1}\left(y_{0}\left(t_{1}^{-}\right)\right), & \text {if } t \in\left(t_{1}, t_{2}\right]\end{cases}
$$

Then $\widehat{\phi}_{t_{1}}$ is an element of $\mathcal{D}_{t_{1}}^{*}$ and $\widehat{\phi}_{t_{1}}=y_{0}$.

Let $y(t)=z(t)+\widehat{\phi}(t), t_{1}<t \leq t_{2}$

Obviously, if $y$ satisfies the integral equation

$$
y(t)=y_{0}\left(t_{1}^{-}\right)+S\left(t-t_{1}\right) I_{1}\left(y_{0}\left(t_{1}^{-}\right)\right)+\int_{t_{1}}^{t} S(t-s) f(s) d s+\int_{t_{1}}^{t} S(t-s) g(s) d B_{Q}^{H}(s), t \in\left[t_{1}, t_{2}\right],
$$

that $z$ satisfies $z\left(t_{1}\right)=0, t \in\left(-\infty, t_{1}\right]$ and

$z(t)=\int_{t_{1}}^{t} S(t-s) f(s) d s+\int_{t_{1}}^{t} S(t-s) g(s) d B_{Q}^{H}(s)+S\left(t-t_{1}\right) I_{1}\left(z_{0}\left(t_{1}^{-}\right)+\widehat{\phi}\left(t_{1}^{-}\right)\right), \quad t \in\left[t_{1}, t_{2}\right]$.

Set $\mathcal{D}_{t_{1}}^{1}=\left\{z \in \mathcal{D}_{t_{1}}^{*}, \quad\right.$ such that $\left.z_{t_{1}}=0\right\}$

Let the operator $\widehat{\Phi}: \mathcal{D}_{t_{1}}^{1} \rightarrow \mathcal{P}\left(\mathcal{D}_{t_{1}}^{1}\right)$ defined by

$$
\widehat{\Phi}(z)=\left\{\widehat{\rho} \in \mathcal{D}_{t_{1}}^{1}: \widehat{\rho}(t)=\left\{\begin{array}{ll}
0, & \text { if } ; \in\left(-\infty, t_{1}\right], \\
\int_{t_{1}}^{t} S(t-s) f(s) d s+\int_{t_{1}}^{t} S(t-s) g(s) d B^{H}(s) & \\
+S\left(t-t_{1}\right)\left(I_{1}\left(z\left(t_{1}^{-}\right)+\phi\left(t_{1}^{-}\right)\right)\right. & \text {if } t \in\left[t_{1}, t_{2}\right] .
\end{array}\right\}\right.
$$


where

$$
f \in N_{F, z+\widehat{\phi}}=\left\{f \in L^{2}\left(\left[t_{1}, t_{2}\right], \mathcal{H}\right): f(t) \in F\left(t, z_{t_{1}}+\widehat{\phi}_{t_{1}}\right) \text { a.e.t } \in\left[t_{1}, t_{2}\right]\right\} .
$$

Similar to Step 1, we can prove that the problem (3.18) has at least one solution, which we denote by $y_{1}$.

Step3. We continue this process and take into account that $z_{m}:=\left.z\right|_{\in\left[t_{m}, T\right]}$ is a fixed point of the operator $\widehat{\Phi}_{m}$ defined by

Let the operator $\widehat{\Phi}: \mathcal{D}_{t_{m}}^{m} \rightarrow \mathcal{P}\left(\mathcal{D}_{t_{m}}^{m}\right)$ defined by

$$
\widehat{\Phi}(z)=\left\{\widehat{\rho} \in \mathcal{D}_{t_{m}}^{m} \widehat{\rho}(t)=\left\{\begin{array}{ll}
0, & \text { if; } t \in\left(-\infty, t_{m}\right], \\
\int_{t_{m}}^{t} S(t-s) f(s) d s+\int_{t_{m}}^{t} S(t-s) g(s) d B^{H}(s) & \\
+S\left(t-t_{m}\right)\left(I_{m}\left(z_{m-1}\left(t_{m}^{-}\right)+\phi\left(t_{m}^{-}\right)\right)\right. & \text {if } t \in\left[t_{m}, T\right] .
\end{array}\right\}\right.
$$

where

$$
f \in N_{z+\widehat{\phi}}=\left\{f \in L^{2}\left(\left[t_{m}, T\right], \mathcal{H}\right): f(t) \in F\left(t, z_{t_{t}}+\widehat{\phi}_{t}\right) \text { a.e.t } \in\left[t_{m}, T\right]\right\} .
$$

Let

$$
\left.\mathcal{D}_{t_{m}}=\left\{z \in\left(\left[t_{m}, T\right], \mathcal{H}\right), z\left(t_{m}^{+}\right) \text {existe } \sup _{t \in\left[t_{m}, T\right]} E\left(|y(t)|^{2}\right)<\infty\right)\right\}
$$

Set

$$
\mathcal{D}_{t_{m}}^{*}=\mathcal{D} \cap \mathcal{D}_{m-1} \cap \mathcal{D}_{m}
$$

and $\mathcal{D}_{t_{m}}^{m}=\left\{z \in \mathcal{D}_{t_{m}}^{*}, \quad\right.$ such that $\left.z_{t_{m}}=0\right\}$

Then, there exists a fixed point $z_{m}$ of $\widehat{\Phi}_{m}$, The fixed point $z$ of the operator $\widehat{\Phi}$ is then defined by

$$
z(t)= \begin{cases}z_{0}(t), & \text { if } t \in\left(-\infty, t_{1}\right], \\ z_{1}(t), & \text { if } t \in\left(t_{1}, t_{2}\right], \\ \cdot & \\ \cdot & \\ z_{m}(t), & \text { if } t \in\left(t_{m}, t_{m+1}\right],\end{cases}
$$

has at least one solution, which we denote by

$$
y(t)= \begin{cases}y_{0}(t), & \text { if } t \in\left(-\infty, t_{1}\right], \\ y_{1}(t), & \text { if } t \in\left(t_{1}, t_{2}\right], \\ \cdot & \\ \cdot & \\ y_{m}(t), & \text { if } t \in\left(t_{m}, t_{m+1}\right],\end{cases}
$$

\section{REFERENCES}

[1] N. U. Ahmed, Nonlinear stochastic differential inclusions on Bananch space, Stochastic Anal. Appl. 12 (1994), 1-10.

[2] N. U. Ahmed, Existence of solutions of nonlinear stochastic differential inclusions on Banach space, in: V. Lakshmikantham (Ed.), Proceedings of the First World Congress of Nonlinear Analysis, Tampa, FL, August (1992) 19-26, , de Gruyter, Berlin, (1996), pp. 1699-1712.

[3] E. Alos, O. Mazet and D. Nualart, Stochastic calculus with respect to Gaussian processes. Ann Probab, 29 (1999), 766-801. 
[4] P. Balasubramaniam, Existence of solutions of functional stochastic differential inclusions, Tamkang J. Math. 33 (2002), 35-43.

[5] P. Balasubramaniam, S. K. Ntouyas and D. Vinayagam, Existence of solutions of semilinear stochastic delay evolution inclusions in a Hilbert space. J. Math. Anal. Appl. 305 (2005), 438-451.

[6] P. Balasubramaniam and D. Vinayagam, Existence of Solutions of Nonlinear Neutral Stochastic Differential Inclusions in a Hilbert Space. Stochastic Analysis and Applications, 23 (2005), 137-151.

[7] D.D. Bainov and P.S. Simeonov, Systems with Impulsive Effect, Horwood, Chichester, 1989.

[8] M. Benchohra, J. Henderson and S.K. Ntouyas, Impulsive Differential Equations and Inclusions, vol. 2, Hindawi Publishing Corporation, New York, 2006.

[9] A. Bielecki, Une remarque sur la mtode de Banach-Cacciopoli-Tikhonov dans la thorie des quations diffrentielles ordinaires, Bull. Acad. Polon. Sci. 4 (1956), 261-264.

[10] A. Bressan and G. Colombo, Extensions and selections of maps with decomposable values, Studia Math. 90 (1988), 69-86.

[11] A. Boudaoui, T. Caraballo and A. Ouahab, Existence of mild solutions to stochastic delay evolution equations with a fractional Brownian motion and impulses, Stoch. Anal. Appl. 33 (2015), 244-258.

[12] B. Boufoussi and S. Hajji Neutral stochastic functional differential equations driven by a fractional Brownian motion in a Hilbert space. Statist Probab Lett, 82 (2012), 1549-1558.

[13] T. Caraballo and D. Mamadou Abdoul, Neutral stochastic delay partial functional integrodifferential equations driven by a fractional Brownian motion Front. Math. China 8 (2013), 745-760.

[14] S. Djebali, L. Gorniewicz and A. Ouahab, Solutions Sets for Differential Equations and Inclusions, De Gruyter Series in Nonlinear Analysis and Applications 18. Berlin: de Gruyter, 2013.

[15] J. Dugundji and A. Granas, Fixed Point Theory, Springer-Verlag, New York, 2003.

[16] K. Deimling, Multivalued Differential Equations, Walter De Gruyter, Berlin-New York, 1992.

[17] M. Frigon and A. Granas: Théorèmes d'existence pour des inclusions différentielles sans convexit, C. R. Acad. Sci. Paris, Ser. 1310 (1990), 819-822.

[18] J. R. Graef, J. Henderson and A. Ouahab, Impulsive differential inclusions. A fixed point approach. De Gruyter Series in Nonlinear Analysis and Applications 20. Berlin: de Gruyter, 2013.

[19] J. K. Hale and J. Kato, Phase space for retarded equations with infinite delay, Funkcial. Ekvac., 21 (1978), 11-41.

[20] Y. Hino, S. Murakami and T. Naito, Functional Differential Equations with Infinite Delay, in: Lecture Notes in Mathematics, vol. 1473, Springer-Verlag, Berlin, 1991.

[21] M. Kisielewicz, Differential Inclusions and Optimal Control, Kluwer, Dordrecht 1991.

[22] V.Lakshmikantham, D.D. Bainov and P.S. Simeonov, Theory of Impulsive Differential Equations. World Scientific, Singapore 1989.

[23] A. Lasota and Z. Opial, An application of the Kakutani-Ky Fan theorem in the theory of ordinary differential equations, Bull. Acad. Pol. Sci. Ser. Sci. Math.Astronom. Phys. 13 (1965), 781-786.

[24] Y. Mishura, Stochastic Calculus for Fractional Brownian Motion and Related Topics. Lecture Notes in Mathematics, Vol 1929. Berlin: Springer-Verlag, 2008.

[25] H. W. Ning and B. Liu, Existence results for impulsive neutral stochastic evolution inclusions in Hilbert Space, Acta Mathematica Sinica, 27 (2011), 1405-1418.

[26] D. Nualart The Malliavin Calculus and Related Topics. 2nd ed. Berlin: Springer- Verlag, 2006 .

[27] S. Tindel, C. Tudor and F. Viens, Stochastic evolution equations with fractional Brownian motion. Probab Theory Related Fields,127 (2003), 186-204.

[28] A.M. Samoilenko and N.A. Perestyuk, Impulsive Differential Equations. World Scientific, Singapore 1995.

[29] A. Pazy, Semigroups of Linear Operators and Applications to Partial Differential Equations. Springer-Verlag, New York, 1983. 
Ahmed Boudaoui

Laboratory of Mathematics, Univ Sidi Bel Abbes

PoBox 89, 22000 Sidi-Bel-Abbes, Algeria.

E-mail address: ahmedboudaoui@yahoo.fr

TOMÁs Caraballo

Depto. Ecuaciones Diferenciales y Análisis Numérico,

Universidad de Sevilla, Campus de Reina Mercedes

41012-SEVIlla, Spain

E-mail address: caraball@us.es

Abdelghani Ouahab

Laboratory of Mathematics, Univ Sidi Bel Abbes

PoBox 89, 22000 Sidi-Bel-AbBes, Algeria.

E-mail address: agh_ouahab@yahoo.fr 